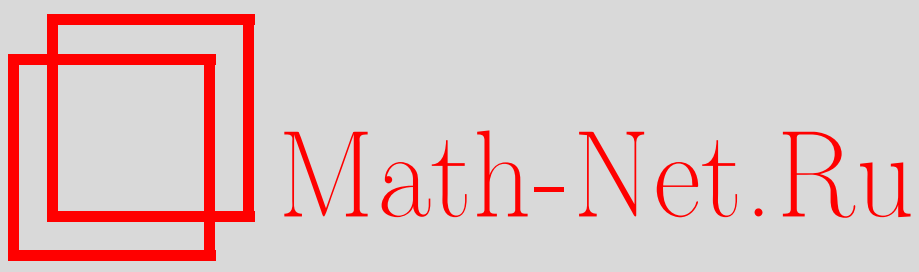

С. А. Голопуз, Определяющие граничные условия и вырожденная задача для эллиптических краевых задач с малым параметром при старших производных, $M a-$ mем. сб., 2003, том 194, номер 5, 3-30

DOI: https://doi.org/10.4213/sm733

Использование Общероссийского математического портала Math-Net.Ru подразумевает, что вы прочитали и согласны с пользовательским соглашением

http://www . mathnet.ru/rus/agreement

Параметры загрузки:

IP: 35.173 .219 .12

26 апреля 2023 г., $17: 25: 30$ 


\author{
С. А. Голопуз
}

\title{
Определяющие граничные условия и вырожденная задача для эллиптических краевых задач с малым параметром при старших производных
}

Рассматривается краевая задача для эллиптического уравнения с мальм параметром при старших производных, когда среди порядков последних $p$ граничных условий имеются сравнимые по модулю $2 p$ (где $2 p$ есть разность между порядками возмущенного и невозмущенного уравнений). Для случая, когда никакие три из них не сравнимы между собой по модулю $2 p$, получены присоединенные граничные условия и доказаны теоремы об асимптотическом разложении.

Библиографол: 6 названий.

\section{§1. Общая постановка задачи}

Пусть $\Omega$ - ограниченная область в $\mathbb{R}^{n}, n \geqslant 1$, с гладкой (класса $C^{\infty}$ ) границей $\Gamma$,

$$
A=\sum_{|\alpha| \leqslant 2 m_{1}} a_{\alpha}(x) \frac{\partial^{\alpha}}{\partial x^{\alpha}}, \quad L=\sum_{|\alpha| \leqslant 2 m_{0}} l_{\alpha}(x) \frac{\partial^{\alpha}}{\partial x^{\alpha}}, \quad m_{1}>m_{0},
$$

- дифференциальные операторы с вешественными коэффициентами класса $C^{\infty}(\bar{\Omega})$, причем

$$
\sum_{|\alpha|=2 m_{1}} a_{\alpha}(x) \xi^{\alpha}>0, \quad \sum_{|\alpha|=2 m_{0}} l_{\alpha}(x) \xi^{\alpha}>0, \quad x \in \bar{\Omega}, \quad \xi \in \mathbb{R}^{n}, \xi \neq 0 .
$$

Пусть также в полуокрестности Г задана нормальная [1] система линейных дифференциальных операторов $B_{j}, j=1,2, \ldots, m_{1}$, с бесконечно дифференцируемыми коэффициентами, причем

$$
r_{1}<r_{2}<\cdots<r_{m_{1}} \leqslant 2 m_{1}-1,
$$

где $r_{j}$ обозначает порядок оператора $B_{j}$. Кроме того, для краткости будем использовать обозначение $p=m_{1}-m_{0}$.

Рассмотрим в области $\Omega$ следуюшую краевую задачу:

$$
\begin{gathered}
L_{\varepsilon} u_{\varepsilon} \equiv \varepsilon^{2 p} A u_{\varepsilon}+(-1)^{p} L u_{\varepsilon}=h, \\
\left.B_{j} u_{\varepsilon}\right|_{\Gamma}=f_{j}, \quad j=1,2, \ldots, m_{1} .
\end{gathered}
$$

Здесь $\varepsilon>0$ - малый параметр, $h \in C^{\infty}(\bar{\Omega}), f_{j} \in C^{\infty}(\Gamma)$. В силу (1.1) вырождение задачи (1.2), (1.3) регулярно [2]. 
ЗАмечаниЕ. Если $n=1$, то $\Omega=(a, b), \Gamma=\{a, b\}$. Тогда (1.2), (1.3) представляет собой краевую задачу для обыкновенного дифференциального уравнения. В этом случае условие нормальности системы $\left\{B_{j}\right\}$ означает, что операторы $\left.B_{j}\right|_{x=a}$ и $\left.B_{j}\right|_{x=b}$ (вообще говоря, различные) имеют одинаковый порядок $r_{j}$.

Предположим, что при $\varepsilon \rightarrow 0$ решение задачи $(1.2),(1.3)$ стремится (в некотором функциональном пространстве) к предельной функции $w_{0}$. Тогда естественно ожидать, что $w_{0}$ будет удовлетворять уравнению (1.2) при $\varepsilon=0$ :

$$
(-1)^{p} L w_{0}=h .
$$

Ясно, что при этом функция $w_{0}$, вообше говоря, не будет удовлетворять всем $m_{1}$ граничньм условиям (1.3). Так как оператор $L$ имеет порядок $2 m_{0}$, то $w_{0}$ может удовлетворять лишь $m_{0}$ независимым граничньм условиям.

Известно, что, например, для первой краевой задачи $\left(B_{j}=\partial^{j-1} / \partial \nu^{j-1}\right)$ таковыми являются первые $m_{0}$ граничных условий (1.3):

$$
\left.B_{j} w_{0}\right|_{\Gamma}=f_{j}, \quad j=1, \ldots, m_{0} .
$$

Однако в общем случае это не всегда так, что и показывает следующий пример краевой задачи на отрезке $[0,1]$ :

$$
\begin{gathered}
\varepsilon^{4} u_{\varepsilon}^{(8)}+4 u_{\varepsilon}^{(4)}=0, \\
u_{\varepsilon}(0)=u_{\varepsilon}(1)=0, \quad u_{\varepsilon}^{\prime}(0)=u_{\varepsilon}^{\prime}(1)=0, \\
u_{\varepsilon}^{\prime \prime}(0)=u_{\varepsilon}^{\prime \prime}(1)=1, \quad u_{\varepsilon}^{(6)}(0)=u_{\varepsilon}^{(6)}(1)=0 .
\end{gathered}
$$

Решение задачи (1.6) задается формулой

$$
\begin{aligned}
u_{\varepsilon}(x)= & \frac{x^{2}-x}{2}+\frac{\varepsilon}{2}\left(\sin ^{2} \frac{1}{\varepsilon}-\operatorname{sh}^{2} \frac{1}{\varepsilon}+2 \varepsilon\left(\operatorname{ch} \frac{1}{\varepsilon}-\cos \frac{1}{\varepsilon}\right)\left(\operatorname{sh} \frac{1}{\varepsilon}-\sin \frac{1}{\varepsilon}\right)\right)^{-1} \\
& \times\left(\left(\operatorname{sh} \frac{1}{\varepsilon}+\sin \frac{1}{\varepsilon}\right)\left(\operatorname{ch} \frac{x}{\varepsilon} \cos \frac{1-x}{\varepsilon}+\cos \frac{x}{\varepsilon} \operatorname{ch} \frac{1-x}{\varepsilon}-\operatorname{ch} \frac{1}{\varepsilon}-\cos \frac{1}{\varepsilon}\right)\right. \\
& +2 \varepsilon\left(\operatorname{sh}^{2} \frac{1}{\varepsilon}+\sin ^{2} \frac{1}{\varepsilon}-\operatorname{sh} \frac{1}{\varepsilon}\left(\operatorname{sh} \frac{x}{\varepsilon} \cos \frac{1-x}{\varepsilon}+\cos \frac{x}{\varepsilon} \operatorname{sh} \frac{1-x}{\varepsilon}\right)\right. \\
& \left.\left.-\sin \frac{1}{\varepsilon}\left(\operatorname{ch} \frac{x}{\varepsilon} \sin \frac{1-x}{\varepsilon}+\sin \frac{x}{\varepsilon} \operatorname{ch} \frac{1-x}{\varepsilon}\right)\right)\right),
\end{aligned}
$$

из которой видно, что $u_{\varepsilon}(x)$ стремится к $\left(x^{2}-x\right) / 2$ равномерно на $[0,1]$ и локально равномерно вместе со всеми производньми на $(0,1)$. А предельная функция $w_{0}=$ $\left(x^{2}-x\right) / 2$ удовлетворяет 1 -му и 3 -му граничным условиям, но не удовлетворяет 2-му граничному условию.

Таким образом, именно 1-е и 3-е граничные условия определяют поведение решения задачи (1.6) при $\varepsilon \rightarrow 0$. Такие граничные условия будем называть определяющими. Точная формулировка этого понятия будет дана в 22 .

В связи с этим возникает вопрос о нахождении граничных условий, которым a priori должна удовлетворять предельная функция (если такая сушествует) для решения задачи (1.2), (1.3). В следуюшем параграфе излагается метод, позволяющий формально получить эти граничные условия (они получат название присоединенных). 


\section{§ 2. Определяющие граничные условия и вырожденная краевая задача}

2.1. Предлагаемый способ выявления определяющих граничных условий задачи (1.2), (1.3) основан на методе Вишика-Люстерника построения асимптотического разложения решений эллиптических краевых задач с малым параметром при старших производных.

Согласно этому методу будем искать решение задачи (1.2), (1.3) в виде асимптотического представления

$$
u_{\varepsilon} \sim \sum_{k=0}^{\infty} \varepsilon^{k}\left(w_{k}+\varepsilon^{k_{0}} v_{k}^{(\varepsilon)}\right)
$$

где $v_{k}^{(\varepsilon)}$ - функция типа пограничного слоя [2], а $w_{k}$ не зависит от $\varepsilon$. Коэффициенты разложения (2.1) предполагаются бесконечно дифференцируемыми в $\bar{\Omega}$ функциями.

Формальная подстановка (2.1) в (1.2) приводит (поскольку внутри $\Omega$ функции $v_{k}^{(\varepsilon)}$ стремятся к нулю быстрее любой степени $\left.\varepsilon\right)$ к системе соотношений

$$
(-1)^{p} L w_{0}=h, \quad(-1)^{p} L w_{k}=-A w_{k-2 p}, \quad k=1,2, \ldots
$$

(здесь и далее полагаем $w_{k}=v_{k}^{(\varepsilon)} \equiv 0$ при $k<0$ ).

Выведем теперь соотношения между функциями пограничного слоя. С этой целью, следуя [2], введем в полуокрестности $\Gamma$ систему координат $(\rho, s)$, где $\rho$ обозначает расстояние до $\Gamma$, а $s$ - точка многообразия $Г$. В этих координатах $Г$ описывается уравнением $\rho=0$, а линии $s=$ const являются отрезками внутренних нормалей к Г. При достаточно малом $\rho_{0}$ отображение $x \mapsto(\rho(x), s(x))$ множества $\left\{x \in \bar{\Omega}: \rho(x)<\rho_{0}\right\}$ на $\left[0, \rho_{0}\right) \times \Gamma$ является $C^{\infty}$-диффеоморфизмом.

Положим $t=\rho / \varepsilon$. В координатах $(t, s)$ при любом $N \in \mathbb{N}$ имеет место представление

$$
L_{\varepsilon}=\varepsilon^{-2 m_{0}}\left(M_{0}+\varepsilon M_{1}+\cdots+\varepsilon^{N} M_{N}+R_{N, \varepsilon}\right),
$$

где операторы $M_{0}, M_{1}, \ldots, M_{N}$ не зависят от $\varepsilon$, а коэффициенты оператора $R_{N, \varepsilon}$ имеют порядок малости $\varepsilon^{N+1}$. В частности, $M_{0}$ и $M_{1}$ имеют вид

$$
\begin{gathered}
M_{0}=a(s) \frac{\partial^{2 m_{1}}}{\partial t^{2 m_{1}}}+(-1)^{p} l(s) \frac{\partial^{2 m_{0}}}{\partial t^{2 m_{0}}} \\
M_{1}=A_{1} \frac{\partial^{2 m_{1}-1}}{\partial t^{2 m_{1}-1}}+(-1)^{p} L_{1} \frac{\partial^{2 m_{0}-1}}{\partial t^{2 m_{0}-1}}+t\left(a_{1}(s) \frac{\partial^{2 m_{1}}}{\partial t^{2 m_{1}}}+(-1)^{p} l_{1}(s) \frac{\partial^{2 m_{0}}}{\partial t^{2 m_{0}}}\right)
\end{gathered}
$$

где $A_{1}$ и $L_{1}$ - дифференциальные операторы на многообразии Г, порядки которых меньше или равны 1 . Из предположений, сделанных в $\S 1$, следует, что коэффициенты операторов $M_{0}, M_{1}, \ldots, M_{N}, R_{N, \varepsilon}$ являются функциями класса $C^{\infty}(\Gamma)$. При этом в силу (1.1)

$$
a(s)>0, \quad l(s)>0
$$

при всех $s \in \Gamma$. 
ЗАмечАнИЕ. В случае $n=1$ граница $Г$ есть многообразие размерности 0, в силу чего $A_{1}$ и $L_{1}$ имеют нулевой порядок.

Полагая $v_{k}^{(\varepsilon)}=v_{k}(t, s)$, приходим согласно [3] к следующим соотношениям:

$$
\begin{gathered}
M_{0} v_{0}=0 \\
M_{0} v_{k}=-\sum_{j=1}^{k} M_{j} v_{k-j}, \quad k=1,2, \ldots .
\end{gathered}
$$

Кроме того, поскольку $v_{k}^{(\varepsilon)}$ является функцией погранслоя, имеем условие на бесконечности

$$
\left.v_{k}\right|_{t=+\infty}=0, \quad k=0,1,2, \ldots .
$$

2.2. Теперь найдем соотношения между коэффициентами разложения (2.1) на гранище области.

Аналогично (2.3) для $B_{j}$ имеет место представление вида

$$
\left.B_{j}\right|_{\Gamma}=\left.\varepsilon^{-r_{j}}\left(b_{j}(s) \frac{\partial^{r_{j}}}{\partial t^{r_{j}}}+\sum_{m=1}^{r_{j}} \varepsilon^{m} B_{j m} \frac{\partial^{r_{j}-m}}{\partial t^{r_{j}-m}}\right)\right|_{t=0}, \quad j=1,2, \ldots, m_{1},
$$

где $B_{j m}$ - дифференциальный оператор на многообразии Г порядка меньше или равного $m$. При этом $b_{j}(s)$ и коэффициенты операторов $B_{j m}$ являются функциями класса $C^{\infty}(\Gamma)$. Если $n=1$, то операторы $B_{j m}=b_{j m}(s)$ будут просто функциями на двухточечном многообразии $Г$.

Подставим (2.1), (2.10) в (1.3) и сгруппируем коэффициенты при одинаковых степенях $\varepsilon$ :

$$
\begin{gathered}
\sum_{k=k_{j}}^{\infty} \varepsilon^{k}\left(\left.B_{j} w_{k}\right|_{\Gamma}+\left.\left(b_{j} \frac{\partial^{r_{j}} v_{k-k_{0}+r_{j}}}{\partial t^{r_{j}}}+\sum_{m=1}^{r_{j}} B_{j m} \frac{\partial^{r_{j}-m} v_{k-k_{0}+r_{j}-m}}{\partial t^{r_{j}-m}}\right)\right|_{t=0}\right) \sim f_{j}, \\
j=1,2, \ldots, m_{1} .
\end{gathered}
$$

Здесь мы ввели обозначения

$$
k_{j}=\min \left\{0, k_{0}-r_{j}\right\}, \quad j=1,2, \ldots, m_{1} .
$$

Отсюда вытекают следуюшие соотношения:

$$
\begin{aligned}
\left.B_{j} w_{k+k_{j}}\right|_{\Gamma} & +\left.\left(b_{j} \frac{\partial^{r_{j}} v_{k+k_{j}-k_{0}+r_{j}}}{\partial t^{r_{j}}}+\sum_{m=1}^{r_{j}} B_{j m} \frac{\partial^{r_{j}-m} v_{k+k_{j}-k_{0}+r_{j}-m}}{\partial t^{r_{j}-m}}\right)\right|_{t=0} \\
& =\delta\left(k+k_{j}\right) f_{j}, \quad j=1,2, \ldots, m_{1}, \quad k=0,1,2, \ldots
\end{aligned}
$$

где

$$
\delta(k)= \begin{cases}0, & k \neq 0 \\ 1, & k=0\end{cases}
$$

Отметим, что в силу нормальности операторов $B_{j}$ функции $b_{j}$ нигде не обращаются в нуль:

$$
b_{j}(s) \neq 0, \quad s \in \Gamma, \quad j=1,2, \ldots, m_{1} .
$$

Таким образом, приходим к выводу, что для того чтобы при заданном $k_{0}$ решение задачи (1.2), (1.3) допускало асимптотическое представление в виде (2.1), необходимо, чтобы была разрешимой система соотношений $(2.2),(2.7)-(2.9),(2.11)$. 
2.3. Выделим из бесконечной системы граничных соотношений (2.11) ту часть (назовем ее усеченной системой), которая соответствует значениям $k \leqslant-k_{j}$, $j=1,2, \ldots, m_{1}$. Тогда из коэффициентов регулярной части разложения $(2.1)$ в усеченную систему будет входить только функция $w_{0}$. Очевидно, что усеченная система зависит от выбора показателя $k_{0}$ в асимптотическом представлении (2.1).

Прежде всего, показатель $k_{0}$ необходимо выбрать так, чтобы выполнялось условие совместности:

а) при любых $w_{0} \in C^{\infty}(\bar{\Omega}), f_{j} \in C^{\infty}(\Gamma)$ усеченная система в совокупности $\mathrm{c}(2.7)$ и $(2.8)$ имеет решение относительно $v_{0}(t, s)$.

Если это условие выполнено, то, исключив (с помощью (2.7) и (2.8)) функции погранслоя из максимально возможного числа соотношений усеченной системы, получим некоторый набор независимых граничных условий на функцию $w_{0}$. Поскольку $w_{0}$ должна быть решением эллиптического уравнения (1.4) порядка $2 m_{0}$, приходим к условию

b) количество граничных условий в полученном наборе в точности равно $m_{0}$.

Предположим, что показатель $k_{0}$ удовлетворяет условиям а) и b). Тогда полученные в результате вышеописанной процедуры граничные условия на $w_{0}$ будем называть присоединенными (к невозмущенному уравнению (1.4)). Ясно, что присоединенные граничные условия задачи $(1.2),(1.3)$ являются следствиями граничных условий (1.3).

ОПРЕДЕЛЕНИЕ 1. Вырожденной краевой задачей для задачи (1.2), (1.3) называется невозмушенное уравнение (1.4) в совокупности с присоединенными граничными условиями.

ОПРЕДЕЛЕНИЕ 2. Определяющими граничными условиями задачи (1.2), (1.3) называются граничные условия (из числа условий (1.3)), у которых задающие их граничные операторы формально входят в присоединенные условия.

Формулировки этих определений основаны на понятии присоединенных граничных условий, способ получения которых описан пока лишь в самых общих чертах. Ясно, однако, что присоединенное граничное условие либо совпадает с одним из определяющих, либо является некоторой их комбинацией.

\section{§3. Присоединенные граничные условия} в случае $r_{j} \not \equiv r_{k}(\bmod 2 p)$ при $m_{0}<j<k \leqslant m_{1}$

3.1. Придадим теперь предыдущим общим рассуждениям конкретный характер.

Прежде всего определим, в каком диапазоне может находиться значение показателя $k_{0}$. Если взять $k_{0}>r_{m_{0}+1}$, то из (2.11) получим, что

$$
\left.B_{j} w_{0}\right|_{\Gamma}=f_{j}, \quad j=1,2, \ldots, m_{0}+1 .
$$

Но вместе с (1.4) это дает переопределенную краевую задачу (не выполняется условие b)). Следовательно, должно быть $k_{0} \leqslant r_{m_{0}+1}$. В этом параграфе будет получен критерий выполнения условия совместности для $k_{0}=r_{m_{0}+1}$. 
3.2. Итак, пусть $k_{0}=r_{m_{0}+1}$. Тогда

$$
k_{j}= \begin{cases}0, & j \leqslant m_{0}+1 \\ r_{m_{0}+1}-r_{j}, & j>m_{0}+1\end{cases}
$$

Поэтому соотношения (2.11) при $k=0$ запишутся следуюшим образом:

$$
\begin{gathered}
\left.B_{j} w_{0}\right|_{\Gamma}=f_{j}, \quad j=1, \ldots, m_{0}, \\
\left.B_{m_{0}+1} w_{0}\right|_{\Gamma}+\left.b_{m_{0}+1} \frac{\partial^{r_{m_{0}+1}} v_{0}}{\partial t^{r_{m_{0}}+1}}\right|_{t=0}=f_{m_{0}+1}, \\
\left.b_{j} \frac{\partial^{r_{j}} v_{0}}{\partial t^{r_{j}}}\right|_{t=0}=0, \quad j=m_{0}+2, \ldots, m_{1} .
\end{gathered}
$$

Далее, следуя обозначениям, введенным в (2.4), запишем уравнение (2.7) в развернутом виде:

$$
a(s) \frac{\partial^{2 m_{1}} v_{0}}{\partial t^{2 m_{1}}}+(-1)^{p} l(s) \frac{\partial^{2 m_{0}} v_{0}}{\partial t^{2 m_{0}}}=0 .
$$

Всякое его решение, удовлетворяющее условию (2.9), имеет вид

$$
v_{0}(t, s)=\sum_{k=1}^{p} C_{0}^{(k)}(s) e^{\lambda_{k}(s) t}
$$

где $\lambda_{1}(s), \ldots, \lambda_{p}(s)$ - корни характеристического уравнения

$$
a(s) \lambda^{2 m_{1}}(s)+(-1)^{p} \lambda^{2 m_{0}}(s)=0
$$

с отрицательными вешественными частями. В силу (2.6) имеется ровно $p$ таких корней. После подстановки (3.3) в (3.2) получается система линейных уравнений

$$
\left\{\begin{array}{l}
\lambda_{1}^{r_{m_{0}+1}} C_{0}^{(1)}+\cdots+\lambda_{p}^{r_{m_{0}+1}} C_{0}^{(p)}=\frac{f_{m_{0}+1}}{b_{m_{0}+1}}-\left.\frac{B_{m_{0}+1}}{b_{m_{0}+1}} w_{0}\right|_{\Gamma} \\
\lambda_{1}^{r_{j}} C_{0}^{(1)}+\cdots+\lambda_{p}^{r_{j}} C_{0}^{(p)}=0, \quad j=m_{0}+2, \ldots, m_{1} .
\end{array}\right.
$$

Таким образом, мы получили $m_{0}$ граничных условий (3.1), совпадаюших с (1.5). Однако присоединенньми (для рассматриваемого значения $k_{0}$ ) они будут лишь при выполнении условия совместности а), т.е. когда определитель системы (3.4)

$$
D\left(r_{m_{0}+1}, \ldots, r_{m_{1}}\right) \equiv\left|\begin{array}{cccc}
\lambda_{1}^{r_{m_{0}+1}} & \lambda_{2}^{r_{m_{0}+1}} & \ldots & \lambda_{p}^{r_{m_{0}+1}} \\
\ldots \ldots \ldots \ldots \ldots \ldots \ldots \ldots . \ldots \\
\lambda_{1}^{r_{m_{1}}} & \lambda_{2}^{r_{m_{1}}} & \ldots & \lambda_{p}^{r_{m_{1}}}
\end{array}\right|
$$

всюду отличен от нуля.

3.3. Выведем критерий равенства нулю определителя системы (3.4). При этом для упрошения записи вместо $r_{m_{0}+1}, \ldots, r_{m_{1}}$ будем соответственно писать $n_{1}, \ldots, n_{p}$. 
ТЕОРема. Определитель $D\left(n_{1}, \ldots, n_{p}\right)$ отличен от нуля в том и только том случае, когда числа $n_{1}, \ldots, n_{p}$ попарно не сравнимы по модулю $2 p$.

ДокАЗАТЕЛЬСтво. Так как функции $\lambda_{1}(s), \ldots, \lambda_{p}(s)$ являются корнями уравнения

$$
a(s) \lambda^{2 p}(s)+(-1)^{p} l(s)=0
$$

с отрицательными вещественньми частями, то

$$
\lambda_{k}=\left(\frac{l}{a}\right)^{1 /(2 p)} e^{(p+2 k-1) \pi i /(2 p)}=\left(\frac{l}{a} e^{(p-1) \pi i}\right)^{1 /(2 p)} e^{k \pi i / p}, \quad k=1,2, \ldots, p
$$

Поэтому

$$
\begin{aligned}
& D\left(n_{1}, \ldots, n_{p}\right)=\left|\begin{array}{llll}
\lambda_{1}^{n_{1}} & \lambda_{2}^{n_{1}} & \ldots & \lambda_{p}^{n_{1}} \\
\ldots \ldots \ldots \ldots \ldots \ldots \\
\lambda_{1}^{n_{p}} & \lambda_{2}^{n_{p}} & \ldots & \lambda_{p}^{n_{p}}
\end{array}\right| \\
& =\left(\frac{l}{a} e^{(p-1) \pi i}\right)^{\left(n_{1}+\cdots+n_{p}\right) /(2 p)}\left|\begin{array}{llll}
e^{n_{1} \pi i / p} & e^{2 n_{1} \pi i / p} & \ldots & e^{n_{1} \pi i} \\
\ldots \ldots \ldots \ldots \ldots \ldots \ldots \ldots & \ldots \ldots \ldots \\
e^{n_{p} \pi i / p} & e^{2 n_{p} \pi i / p} & \ldots & e^{n_{p} \pi i}
\end{array}\right| \\
& =\left(\frac{l}{a} e^{(p-1) \pi i}\right)^{\left(n_{1}+\cdots+n_{p}\right) /(2 p)} \\
& \times e^{\left(n_{1}+\cdots+n_{p}\right) \pi i / p}\left|\begin{array}{cccc}
1 & e^{n_{1} \pi i / p} & \ldots & e^{(p-1) n_{1} \pi i / p} \\
\ldots \ldots \ldots \ldots \ldots \ldots \ldots \ldots \ldots \ldots & \ldots \ldots \ldots \\
1 & e^{n_{p} \pi i / p} & \ldots & e^{(p-1) n_{p} \pi i / p}
\end{array}\right| \\
& =\left(\frac{l}{a} e^{(p+1) \pi i}\right)^{\left(n_{1}+\cdots+n_{p}\right) /(2 p)}\left|\begin{array}{cccc}
1 & \alpha_{1} & \ldots & \alpha_{1}^{p-1} \\
\ldots & \ldots & \ldots & \ldots \\
1 & \alpha_{p} & \ldots & \ldots \\
\alpha_{p}^{p-1}
\end{array}\right|,
\end{aligned}
$$

где $\alpha_{k}=e^{n_{k} \pi i / p}, k=1, \ldots, p$.

Таким образом, определитель системы (3.4) свелся к определителю Вандермонда, который отличен от нуля тогда и только тогда, когда числа $\alpha_{k}$ попарно различны, т.е. когда $n_{j} \not \equiv n_{k}(\bmod 2 p)$ при $j \neq k$, что и требовалось доказать.

Эта теорема, в частности, показывает, что если определитель системы (3.4) обращается в нуль хотя бы в одной точке $s_{0} \in \Gamma$, то он тождественно равен нулю на $\Gamma$.

3.4. Полученные результаты приводят к следующим выводам.

Если $r_{j} \not \equiv r_{k}(\bmod 2 p)$ при $m_{0}+1 \leqslant j<k \leqslant m_{1}$, то граничные условия $(1.5)$ являются присоединенными (и, следовательно, определяющими), а вырожденная задача принимает традиционный вид (1.4), (1.5). Так будет всегда, если $p=1$.

Если среди чисел $r_{m_{0}+1}, \ldots, r_{m_{1}}$ имеются сравнимые по модулю $2 p$, то найти $u_{\varepsilon}$ в виде $(2.1)$ с показателем $k_{0}=r_{m_{0}+1}$ не представляется возможным. Следовательно, в этом случае в разложении (2.1) надо полагать $k_{0}<r_{m_{0}+1}$. В дальнейшем будет установлено, в каких случаях может иметь место представление (2.1) с показателем $k_{0}<r_{m_{0}+1}$. Для $p=2$ этот вопрос изучен в [4]. 


\section{§4. Присоединенные граничные условия в случае сравнимости по модулю $2 p$}

4.1. Пусть $J=\left\{r_{m_{0}+1}, \ldots, r_{m_{1}}\right\}, \pi$ - каноническая сюръекция $\mathbb{Z}$ на $\mathbb{Z}_{2 p}$. В дальнейшем будем использовать обозначения

$$
J_{k}=\left\{j \in J: \operatorname{card}\left(\pi^{-1} \pi j \cap J\right)=k\right\}, \quad q=\operatorname{card}(\pi J) .
$$

Так как $\operatorname{card} J=p$, то $q \leqslant p, J=\bigcup_{k=1}^{p} J_{k}$. Ясно, что число $q$ равно рангу системы (3.4).

Для упрошения дальнейших выкладок впредь будем считать операторы $B_{j}$ нормированными:

$$
b_{j}(s) \equiv 1, \quad j=1,2, \ldots, m_{1} .
$$

В силу (2.12) этого всегда можно добиться, заменив $f_{j}$ на $f_{j} / b_{j}$ и $B_{j}$ на $B_{j} / \widetilde{b}_{j}$, где $\widetilde{b}_{j} \neq 0$ - бесконечно дифференцируемая в полуокрестности Г функция, совпадаюшая с $b_{j}$ на $\Gamma$.

4.2. Итак, пусть $q<p$; положим $k_{0}=r_{m_{0}+1}-1$. Тогда $k_{1}=\cdots=k_{m_{0}}=0$, $k_{m_{0}+1}=-1, k_{j}=r_{m_{0}+1}-r_{j}-1, j=m_{0}+2, \ldots, m_{1}$. Вьпишем с учетом (4.1) начальные (при $k=0$ ) соотношения (2.11):

$$
\begin{gathered}
\left.B_{j} w_{0}\right|_{\Gamma}=f_{j}, \quad j=1, \ldots, m_{0}-1, \\
\left.B_{m_{0}} w_{0}\right|_{\Gamma}+\left.\delta\left(r_{m_{0}}-r_{m_{0}+1}+1\right) \frac{\partial^{r_{m_{0}}} v_{0}}{\partial t^{r_{m_{0}}}}\right|_{t=0}=f_{m_{0}}, \\
\left.\frac{\partial^{r_{j}} v_{0}}{\partial t^{r_{j}}}\right|_{t=0}=0, \quad j=m_{0}+1, \ldots, m_{1} .
\end{gathered}
$$

Используя (3.3), получаем

$$
\begin{gathered}
\left\{\begin{array}{c}
\left.B_{j} w_{0}\right|_{\Gamma}=f_{j}, \quad j=1, \ldots, m_{0}-1 \\
\left.B_{m_{0}} w_{0}\right|_{\Gamma}+\delta\left(r_{m_{0}}-r_{m_{0}+1}+1\right) \sum_{k=1}^{p} \lambda_{k}^{r_{m_{0}}} C_{0}^{(k)}=f_{m_{0}}
\end{array}\right. \\
\sum_{k=1}^{p} \lambda_{k}^{r_{j}} C_{0}^{(k)}=0, \quad j=m_{0}+1, \ldots, m_{1} .
\end{gathered}
$$

Кроме того, (2.11) при $k=1, j=m_{0}+1, \ldots, m_{1}$ дает

$$
\begin{gathered}
\left.B_{m_{0}+1} w_{0}\right|_{\Gamma}+\left.\left(\frac{\partial^{r_{m_{0}+1}} v_{1}}{\partial t^{r_{m_{0}}+1}}+B_{m_{0}+1,1} \frac{\partial^{r_{m_{0}+1}-1} v_{0}}{\partial t^{r_{m_{0}+1}}-1}\right)\right|_{t=0}=f_{m_{0}+1} \\
\left.\left(\frac{\partial^{r_{j}} v_{1}}{\partial t^{r_{j}}}+B_{j 1} \frac{\partial^{r_{j}-1} v_{0}}{\partial t^{r_{j}-1}}\right)\right|_{t=0}=0, \quad j=m_{0}+2, \ldots, m_{1} .
\end{gathered}
$$

Поскольку $q<p$, в системе (4.3) среди чисел $r_{j}$ имеются сравнимые по модулю $2 p$. 
Пусть $m_{0}+1 \leqslant i<j \leqslant m_{1}, r_{i} \equiv r_{j}(\bmod 2 p)$. Тогда $r_{j}=r_{i}+2 p N, N \in \mathbb{N}$. Из (4.4) имеем

$$
\begin{gathered}
\left.\delta\left(i-m_{0}-1\right) B_{m_{0}+1} w_{0}\right|_{\Gamma}+\left.\left(\frac{\partial^{r_{i}} v_{1}}{\partial t^{r_{i}}}+B_{i 1} \frac{\partial^{r_{i}-1} v_{0}}{\partial t^{r_{i}-1}}\right)\right|_{t=0}=\delta\left(i-m_{0}-1\right) f_{m_{0}+1} \\
\left.\left(\frac{\partial^{r_{j}} v_{1}}{\partial t^{r_{j}}}+B_{j 1} \frac{\partial^{r_{j}-1} v_{0}}{\partial t^{r_{j}-1}}\right)\right|_{t=0}=0 .
\end{gathered}
$$

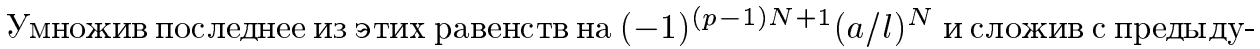
щим, получим

$$
\begin{aligned}
& \left.\delta\left(i-m_{0}-1\right) B_{m_{0}+1} w_{0}\right|_{\Gamma}+\left.\left(\frac{\partial^{r_{i}} v_{1}}{\partial t^{r_{i}}}-(-1)^{(p-1) N}\left(\frac{a}{l}\right)^{N} \frac{\partial^{r_{j}} v_{1}}{\partial t^{r_{j}}}\right)\right|_{t=0} \\
& +\left.\left(B_{i 1} \frac{\partial^{r_{i}-1} v_{0}}{\partial t^{r_{i}-1}}-(-1)^{(p-1) N}\left(\frac{a}{l}\right)^{N} B_{j 1} \frac{\partial^{r_{j}-1} v_{0}}{\partial t^{r_{j}-1}}\right)\right|_{t=0} \\
& \quad=\delta\left(i-m_{0}-1\right) f_{m_{0}+1} .
\end{aligned}
$$

Представим первую скобку в виде

$$
\begin{aligned}
& \frac{\partial^{r_{i}} v_{1}}{\partial t^{r_{i}}}-(-1)^{(p-1) N}\left(\frac{a}{l}\right)^{N} \frac{\partial^{r_{j}} v_{1}}{\partial t^{r_{j}}} \\
& \quad=\sum_{m=0}^{N-1}(-1)^{(p-1) m}\left(\frac{a}{l}\right)^{m}\left(\frac{\partial^{n_{m}} v_{1}}{\partial t^{n_{m}}}+(-1)^{p} \frac{a}{l} \frac{\partial^{n_{m+1}} v_{1}}{\partial t^{n_{m+1}}}\right),
\end{aligned}
$$

где $n_{m}=r_{i}+2 p m$. Так как $n_{m} \leqslant r_{m_{1}}-2 p \leqslant 2 m_{1}-1-2 p=2 m_{0}-1$, то $2 m_{0}-n_{m} \geqslant 1,0 \leqslant m \leqslant N-1$.

Теперь положим в $(2.8) k=1$ и подставим $(2.4),(2.5)$ :

$$
\begin{aligned}
& a \frac{\partial^{2 m_{1}} v_{1}}{\partial t^{2 m_{1}}}+(-1)^{p} l \frac{\partial^{2 m_{0}} v_{1}}{\partial t^{2 m_{0}}} \\
& =-A_{1} \frac{\partial^{2 m_{1}-1} v_{0}}{\partial t^{2 m_{1}-1}}-(-1)^{p} L_{1} \frac{\partial^{2 m_{0}-1} v_{0}}{\partial t^{2 m_{0}-1}} \\
& \quad-t\left(a_{1} \frac{\partial^{2 m_{1}} v_{0}}{\partial t^{2 m_{1}}}+(-1)^{p} l_{1} \frac{\partial^{2 m_{0}} v_{0}}{\partial t^{2 m_{0}}}\right) .
\end{aligned}
$$

Проинтегрируем это уравнение $2 m_{0}-n_{m}$ раз:

$$
\begin{aligned}
a \frac{\partial^{n_{m}+2 p} v_{1}}{\partial t^{n_{m}+2 p}}+(-1)^{p} l \frac{\partial^{n_{m}} v_{1}}{\partial t^{n_{m}}} & \\
= & -A_{1} \frac{\partial^{n_{m}+2 p-1} v_{0}}{\partial t^{n_{m}+2 p-1}}-(-1)^{p} L_{1} \frac{\partial^{n_{m}-1} v_{0}}{\partial t^{n_{m}-1}} \\
& \quad-t\left(a_{1} \frac{\partial^{n_{m}+2 p} v_{0}}{\partial t^{n_{m}+2 p}}+(-1)^{p} l_{1} \frac{\partial^{n_{m}} v_{0}}{\partial t^{n_{m}}}\right) \\
& +\left(2 m_{0}-n_{m}\right)\left(a_{1} \frac{\partial^{n_{m}+2 p-1} v_{0}}{\partial t^{n_{m}+2 p-1}}+(-1)^{p} l_{1} \frac{\partial^{n_{m}-1} v_{0}}{\partial t^{n_{m}-1}}\right)
\end{aligned}
$$


(в силу условия на бесконечности (2.9) все константы, образующиеся при последовательном интегрировании, обратятся в нуль).

Отсюда, поскольку $\lambda_{k}^{2 p}=(-1)^{p-1} l / a$, получаем

$$
\begin{aligned}
& \left.\left(\frac{\partial^{n_{m}} v_{1}}{\partial t^{n_{m}}}+(-1)^{p} \frac{a}{l} \frac{\partial^{n_{m+1}} v_{1}}{\partial t^{n_{m+1}}}\right)\right|_{t=0} \\
& =\left.\left(\frac{\left(2 m_{0}-n_{m}\right) a_{1}-A_{1}}{(-1)^{p} l} \frac{\partial^{n_{m}+2 p-1} v_{0}}{\partial t^{n_{m}+2 p-1}}+\frac{\left(2 m_{0}-n_{m}\right) l_{1}-L_{1}}{l} \frac{\partial^{n_{m}-1} v_{0}}{\partial t^{n_{m}-1}}\right)\right|_{t=0} \\
& =(-1)^{p} \frac{\left(2 m_{0}-n_{m}\right) a_{1}-A_{1}}{l} \sum_{k=1}^{p} \lambda_{k}^{n_{m}+2 p-1} C_{0}^{(k)} \\
& \quad+\frac{\left(2 m_{0}-n_{m}\right) l_{1}-L_{1}}{l} \sum_{k=1}^{p} \lambda_{k}^{n_{m}-1} C_{0}^{(k)} \\
& =\left(\frac{A_{1}}{l} \frac{l}{a}-\frac{L_{1}}{l}+\left(2 m_{0}-n_{m}\right)\left(\frac{l_{1}}{l}-\frac{a_{1}}{a}\right)\right) \sum_{k=1}^{p} \lambda_{k}^{n_{m}-1} C_{0}^{(k)}
\end{aligned}
$$

вследствие чего

$$
\begin{aligned}
\left.\left(\frac{\partial^{r_{i}} v_{1}}{\partial t^{r_{i}}}-(-1)^{(p-1) N}\left(\frac{a}{l}\right)^{N} \frac{\partial^{r_{j}} v_{1}}{\partial t^{r_{j}}}\right)\right|_{t=0} & \\
= & \sum_{m=0}^{N-1}(-1)^{(p-1) m}\left(\frac{a}{l}\right)^{m}\left(\frac{A_{1}}{l} \frac{l}{a}-\frac{L_{1}}{l}-\left(2 m_{0}-n_{m}\right)\left(\frac{a_{1}}{a}-\frac{l_{1}}{l}\right)\right) \\
& \times \sum_{k=1}^{p} \lambda_{k}^{r_{i}+2 p m-1} C_{0}^{(k)} \\
= & \sum_{m=0}^{N-1}(-1)^{(p-1) m}\left(\frac{a}{l}\right)^{m}\left(\frac{A_{1}}{l} \frac{l}{a}-\frac{L_{1}}{l}-\left(2 m_{0}-n_{m}\right)\left(\frac{a_{1}}{a}-\frac{l_{1}}{l}\right)\right) \\
& \times(-1)^{(p-1) m}\left(\frac{l}{a}\right)^{m} \sum_{k=1}^{p} \lambda_{k}^{r_{i}-1} C_{0}^{(k)} \\
= & \sum_{m=0}^{N-1}\left(\left(\frac{a}{l}\right)^{m}\left(\frac{A_{l}}{l} \frac{l}{a}-\frac{L_{1}}{l}\right)\left(\frac{l}{a}\right)^{m}-\left(2 m_{0}-r_{i}-2 p m\right)\left(\frac{a_{1}}{a}-\frac{l_{1}}{l}\right)\right) \\
& \times \sum_{k=1}^{p} \lambda_{k}^{r_{i}-1} C_{0}^{(k)} .
\end{aligned}
$$

\section{Аналогично}

$$
\begin{gathered}
\left.\left(B_{i 1} \frac{\partial^{r_{i}-1} v_{0}}{\partial t^{r_{i}-1}}-(-1)^{(p-1) N}\left(\frac{a}{l}\right)^{N} B_{j 1} \frac{\partial^{r_{j}-1} v_{0}}{\partial t^{r_{j}-1}}\right)\right|_{t=0} \\
=\left(B_{i 1}-\left(\frac{a}{l}\right)^{N} B_{j 1}\left(\frac{l}{a}\right)^{N}\right) \sum_{k=1}^{p} \lambda_{k}^{r_{i}-1} C_{0}^{(k)}
\end{gathered}
$$


Поэтому (4.5) перепишется следующим образом:

$$
\left.\delta\left(i-m_{0}-1\right) B_{m_{0}+1} w_{0}\right|_{\Gamma}-T_{i} \sum_{k=1}^{p} \lambda_{k}^{r_{i}-1} C_{0}^{(k)}=\delta\left(i-m_{0}-1\right) f_{m_{0}+1}
$$

где

$$
\begin{gathered}
T_{i}=\sum_{m=0}^{N-1}\left(\left(\frac{a}{l}\right)^{m}\left(\frac{L_{l}}{l}-\frac{A_{1}}{l} \frac{l}{a}\right)\left(\frac{l}{a}\right)^{m}+\left(2 m_{0}-r_{i}-2 p m\right)\left(\frac{a_{1}}{a}-\frac{l_{1}}{l}\right)\right) \\
-B_{i 1}+\left(\frac{a}{l}\right)^{N} B_{j 1}\left(\frac{l}{a}\right)^{N} .
\end{gathered}
$$

- дифференциальньй оператор не выше первого порядка на компактном многообразии $Г$.

Система (4.3) дает $q$ независимых линейных соотношений между $C_{0}^{(1)}(s), \ldots$, $C_{0}^{(p)}(s)$. Кроме того, предыдущие вычисления позволяют получить еще $p-q$ независимых между собой соотношений вида (4.7). Здесь мы ограничимся рассмотрением случая, когда полученная система $p$ соотношений является полной в том смысле, что число различных (по модулю $2 p$ ) значений показателей $r_{j}$ в $(4.3)$ и $r_{i-1}$ в $(4.7)$ равно $p$. Последнее в точности означает, что

$$
J=J_{1} \cup J_{2}, \quad(\pi J+1) \cap \pi J_{2}=\varnothing,
$$

где $\pi J+1=\{\pi j+1: j \in J\}$.

4.3. Пусть теперь порядки граничных операторов удовлетворяют условию (4.9). Тогда при сужении канонической сюръекции $\pi$ на $J$ каждый элемент из $\mathbb{Z}_{2 p}$ может иметь не более двух прообразов. Поэтому $p \leqslant 2 q, p-q \leqslant q$. Расположим уравнения (4.3) в порядке индексов $\left(i_{1}, \ldots, i_{q}, j_{1}, \ldots, j_{p-q}\right)$ так, чтобы $r_{i_{1}}, \ldots, r_{i_{p-q}} \in J_{2}, i_{1}<\cdots<i_{p-q}, i_{p-q+1}<\cdots<i_{q}, r_{i_{k}} \equiv r_{j_{k}}(\bmod 2 p)$, $i_{k}<j_{k}, k=1, \ldots, p-q$. Тогда первые $q$ уравнений дадут линейно независимую систему

$$
\sum_{m=1}^{p} \lambda_{m}^{r_{i}} C_{0}^{(m)}=0, \quad k=1, \ldots, q
$$

эквивалентную системе (4.3). В свою очередь, вычисления п. 4.2 приводят к системе соотношений

$$
\begin{gathered}
\left.\delta\left(i-m_{0}-1\right) B_{m_{0}+1} w_{0}\right|_{\Gamma}-T_{i_{k}} \sum_{m=1}^{p} \lambda_{m}^{r_{i_{k}}-1} C_{0}^{(m)}=\delta\left(i_{k}-m_{0}-1\right) f_{m_{0}+1} \\
k=1, \ldots, p-q
\end{gathered}
$$

где оператор $T_{i_{k}}$ определяется формулой (4.8), в которой $N=N_{k}=\left(j_{k}-i_{k}\right) /(2 p)$.

В дальнейшем будем исходить из того, что уравнения $T_{i_{k}} g=f, k=1, \ldots, p-q$, имеют (единственное) решение в классе $C^{\infty}(\Gamma)$ при всех $f \in C^{\infty}(\Gamma)$. 
Предположим сначала, что $r_{m_{0}+1} \in J_{1}$. Тогда

$$
\delta\left(i_{1}-m_{0}-1\right)=0, \quad T_{i_{k}} \sum_{m=1}^{p} \lambda_{m}^{r_{i_{k}}-1} C_{0}^{(m)}=0
$$

откуда

$$
\sum_{m=1}^{p} \lambda_{m}^{r_{i_{k}}-1} C_{0}^{(m)}=0, \quad k=1, \ldots, p-q .
$$

Вместе с (4.10) это дает линейную однородную систему $p$ уравнений с $p$ неизвестными. В силу (4.9) ее определитель всюду отличен от нуля и поэтому $C_{0}^{(1)}=\cdots=$ $C_{0}^{(p)} \equiv 0$, т.е. $v_{0}^{(\varepsilon)} \equiv 0$. Но тогда мы приходим к разложению $(2.1)$, в котором $k_{0}=r_{m_{0}+1}$. А это, как было показано в $\S 3$, противоречит условию совместности. Значит, в этом случае решение задачи (1.2), (1.3) надо искать в виде (2.1) с показателем $k_{0} \leqslant r_{m_{0}+1}-2$.

Пусть теперь $r_{m_{0}+1} \in J_{2}$ (откуда $\left.i_{1}=m_{0}+1\right)$. Если при этом $r_{m_{0}+1}=r_{m_{0}}+1$, то из (4.2) и (4.11) вытекает, что

$$
\begin{gathered}
\left.B_{m_{0}} w_{0}\right|_{\Gamma}+\sum_{m=1}^{p} \lambda_{m}^{r_{m_{0}}} C_{0}^{(m)}=f_{m_{0}} \\
\left.B_{m_{0}+1} w_{0}\right|_{\Gamma}-T_{m_{0}+1} \sum_{m=1}^{p} \lambda_{m}^{r_{m_{0}+1}-1} C_{0}^{(m)}=f_{m_{0}+1}
\end{gathered}
$$

Поскольку $r_{m_{0}+1}-1=r_{m_{0}}$, отсюда получаем граничное условие

$$
\left.\left(B_{m_{0}+1}+T_{m_{0}+1} B_{m_{0}}\right) w_{0}\right|_{\Gamma}=f_{m_{0}+1}+T_{m_{0}+1} f_{m_{0}} .
$$

Если же $r_{m_{0}+1}>r_{m_{0}}+1$, то $\delta\left(r_{m_{0}}-r_{m_{0}+1}+1\right)=0$ и (4.2) сводится к $(1.5)$. При этом также имеем

$$
\begin{gathered}
T_{m_{0}+1} \sum_{m=1}^{p} \lambda_{m}^{r_{m_{0}+1}-1} C_{0}^{(m)}=\left.B_{m_{0}+1} w_{0}\right|_{\Gamma}-f_{m_{0}+1}, \\
\sum_{m=1}^{p} \lambda_{m}^{r_{i_{k}-1}} C_{0}^{(m)}=0, \quad k=2, \ldots, p-q .
\end{gathered}
$$

Таким образом, можно сделать окончательный вывод относительно присоединенных граничных условий задачи $(1.2),(1.3)$ в том случае, когда $r_{m_{0}+1} \in J_{2}$ и порядки граничных операторов удовлетворяют условию (4.9).

Если $r_{m_{0}+1}>r_{m_{0}}+1$, то присоединенньми (и как следствие определяющими) являются первые $m_{0}$ граничных условий (1.3). Тогда вырожденная краевая задача имеет традиционный вид (1.4), (1.5). Если же $r_{m_{0}+1}=r_{m_{0}}+1$, то определяющими будут (как правило) первые $m_{0}+1$ граничных условий (1.3). Вырожденной задачей в этом случае является краевая задача

$$
\begin{gathered}
L w_{0}=(-1)^{p} h,\left.\quad B_{j} w_{0}\right|_{\Gamma}=f_{j}, \quad j=1, \ldots, m_{0}-1, \\
\left.\left(B_{m_{0}+1}+T_{m_{0}+1} B_{m_{0}}\right) w_{0}\right|_{\Gamma}=f_{m_{0}+1}+T_{m_{0}+1} f_{m_{0}} .
\end{gathered}
$$


Следует обратить внимание на то, что в последнем случае определяющие граничные условия не являются равноправными, поскольку к $m_{0}$-у граничному условию (1.3) дополнительно применяется оператор $T_{m_{0}+1}$, содержащий дифференцирования лишш по касательным к Г направлениям. Если заменить $\left(m_{0}+1\right)$-е граничное условие (1.3) на

$$
\left.\left(B_{m_{0}+1}+T_{m_{0}+1} B_{m_{0}}\right) u_{\varepsilon}\right|_{\Gamma}=f_{m_{0}+1}+T_{m_{0}+1} f_{m_{0}}
$$

мы получим краевую задачу, эквивалентную задаче (1.2), (1.3). Для нее $\left(m_{0}+1\right)$-е граничное условие уже будет присоединенным, а $m_{0}$-е граничное условие вообще вьпадет из числа определяющих. Именно такая ситуация имеет место для краевой задачи (1.6).

Действительно, согласно сделанным в $\S 2$ замечаниям при $n=1$

$$
A_{1}=a_{0}(s), \quad L_{1}=l_{0}(s), \quad B_{j 1}=b_{j 1}(s) .
$$

Для задачи (1.6) $a_{0}=l_{0}=a_{1}=l_{1}=b_{31}=b_{41}=0$, откуда $T_{3}=0$, в силу чего присоединенными являются 1-е и 3 -е граничные условия.

\section{§5. Теоремы об асимптотическом разложении}

5.1. Докажем теперь теоремы об асимптотическом разложении решения задачи $(1.2),(1.3)$ в случае, когда среди чисел $r_{m_{0}+1}, \ldots, r_{m_{1}}$ имеются сравнимые по модулю $2 p$. Отметим, что при $r_{j} \not \equiv r_{k}(\bmod 2 p), m_{0}+1 \leqslant j<k \leqslant m_{1}$ (т.е. при $J=J_{1}$ ) аналогичная теорема вытекает из результатов, приведенных в [3] (при $n=1)$ и $[5]$.

Для получения нужной оценки остаточного члена будем предполагать, следуя терминологии работы [2], что задача (1.2), (1.3) обладает свойством равномерной разрешимости. Уточним последнее понятие.

Поскольку исходные данные (коэффициенты операторов и граница области) принадлежат классу $C^{\infty}$, целесообразно дать следующие определения.

ОПРЕДЕЛЕНИЕ 3. Будем говорить, что краевая задача разрешима, если при любых правых частях класса $C^{\infty}$ она имеет решение в классе $C^{\infty}(\bar{\Omega})$.

ОПРЕДЕЛЕНИЕ 4. Задача (1.2), (1.3) называется равномерно разрешимой, если существуют натуральное число $k_{0}$ и положительное число $\varepsilon_{0}$, при которых

а) задача (1.2), (1.3) разрешима при $\varepsilon \in\left(0, \varepsilon_{0}\right]$;

б) существуют функциональные пространства с нормами $\|\cdot\|_{0}$ и $\|\cdot\|_{1}$ такие, что для всех $u \in C^{\infty}(\bar{\Omega})$, удовлетворяющих однородным граничным условиям (1.3), выполняется неравенство

$$
\|u\|_{1} \leqslant C \varepsilon^{-k_{0}}\left\|L_{\varepsilon} u\right\|_{0}, \quad 0<\varepsilon \leqslant \varepsilon_{0} .
$$

Если оценка (5.1) имеет место при заданном значении $k_{0}$, будем говорить, что задача равномерно разрешима с показателем $k_{0}$. 
ЗАмЕчАНИЕ. В определении равномерной разрешимости нормы $\|\cdot\|_{0}$ и $\|\cdot\|_{1}$ не фиксируются. Если имеется в виду неравенство (5.1) при конкретных нормах, будем говорить, что задача равномерно разрешима относительно соответствующих им функциональных пространств. В $\$ 6$ будет получен геометрический критерий равномерной разрешимости относительно соболевских пространств $H^{q-2 m_{1}}(\Omega)$ и $H^{q}(\Omega)$, где $q$ - произвольное целое число, $q \geqslant 2 m_{1}$.

Впредь будем считать, что нормы $\|\cdot\|_{0}$ и $\|\cdot\|_{1}$ допускают оценки

$$
\|\cdot\|_{0} \leqslant \text { const }\|\cdot\|_{H^{q_{0}(\Omega)}}, \quad\|\cdot\|_{1} \leqslant \text { const }\|\cdot\|_{H^{q_{1}(\Omega)}},
$$

где $q_{0}$ и $q_{1}-$ неотрицательные целые числа. Этим свойством обладают практически все обшеупотребительные функциональные банаховы пространства.

5.2. ТЕОРЕМА 1. Пусть порядки граничных операторов (1.3) удовлетворяют условию (4.9), причем

1) $r_{m_{0}+1} \in J_{2}, r_{m_{0}+1}=r_{m_{0}}+1$;

2) для всех $f \in C^{\infty}(\Gamma)$ уравнения

$$
T_{i_{k}} g=f
$$

при $k=2, \ldots, p-q$ разрешимы в классе $C^{\infty}(\Gamma)$;

3) вырожденная задача (4.13) разрешима;

4) задача (1.2), (1.3) равномерно разрешима.

Тогда при любом $N \in \mathbb{N} \cup\{0\}$ решение задачи (1.2), (1.3) допускает представление в виде

$$
u_{\varepsilon}=\sum_{k=0}^{N} \varepsilon^{k}\left(w_{k}+\varepsilon^{r_{m_{0}+1}-1} v_{k}^{(\varepsilon)}\right)+z_{N, \varepsilon}
$$

где $w_{k} \in C^{\infty}(\bar{\Omega})$ не зависит от $\varepsilon, v_{k}^{(\varepsilon)} \in C^{\infty}(\bar{\Omega})$ есть функиия пограничного слоя, а остаточный член $z_{N, \varepsilon}$ удовлетворяет оценке

$$
\left\|z_{N, \varepsilon}\right\|_{1} \leqslant C_{N} \varepsilon^{N+\min \left\{1, r_{m_{0}+1}-q_{1}+1 / 2\right\}}, \quad 0<\varepsilon \leqslant \varepsilon_{0} .
$$

ДоказАТЕЛьство. Шаг 1. Начальный шаг итерационного процесса. Прежде всего, условие 3 ) обеспечивает сушествование функции $w_{0} \in C^{\infty}(\bar{\Omega})$, являющейся решением краевой задачи (4.13). Далее, согласно (4.2), (4.10) и (4.12) имеем систему уравнений

$$
\left\{\begin{array}{l}
\sum_{m=1}^{p} \lambda_{m}^{r_{i_{k}}} C_{0}^{(m)}=0, \quad k=1, \ldots, q \\
\sum_{m=1}^{p} \lambda_{m}^{r_{i_{k}}-1} C_{0}^{(m)}=\left.\delta(k-1)\left(f_{m_{0}}-B_{m_{0}} w_{0}\right)\right|_{\Gamma}, \quad k=1, \ldots, p-q
\end{array}\right.
$$

(в силу условия 1) $\left.i_{1}=m_{0}+1\right)$. Из (4.9) вытекает, что ее определитель всюду на Г отличен от нуля. Следовательно, эта система однозначно определяет 
$C_{0}^{(1)}, \ldots, C_{0}^{(p)}$, после чего, исходя из $(3.3)$, умножением на “срезаюшую” функцию класса $C^{\infty}(\bar{\Omega})$, равную единице в полуокрестности Г и нулю вне большей полуокрестности, строим коэффициент $v_{0}^{(\varepsilon)} \in C^{\infty}(\bar{\Omega})$. Кроме того, после подстановки $(3.3)$ в (4.6) находим частное решение $\bar{v}_{1}(t, s)$, которое, очевидно, будет удовлетворять условию (2.9). По построению полученные функции удовлетворяют соотношениям (1.4), (2.7), (2.9) и (2.11) при $k=0$.

Шаг 2. Итерационный процесс. Доказательство существования набора функций, удовлетворяющих всем требуемьм соотношениям, проведем индукцией по $k$. Пусть $w_{0}, \ldots, w_{k-1}, v_{0}^{(\varepsilon)}, \ldots, v_{k-1}^{(\varepsilon)}, k \geqslant 1$, удовлетворяют требуемьм соотношениям. Также будем считать известной функцию $\bar{v}_{k}(t, s)$ - частное решение уравнения (2.8), удовлетворяюшее условию (2.9). Вышеперечисленные функции предполагаются бесконечно дифференцируемыми. Согласно шагу 1 сделанные здесь предположения справедливы при $k=1$.

Прежде всего, имеет место формула

$$
v_{k}(t, s)=\sum_{m=1}^{p} C_{k}^{(m)}(s) e^{\lambda_{m}(s) t}+\bar{v}_{k}(t, s)
$$

Подставив ее в (2.11), получим

$$
\begin{gathered}
\left.B_{j} w_{k}\right|_{\Gamma}+\delta\left(j-m_{0}\right) \sum_{m=1}^{p} \lambda_{m}^{r_{j}} C_{k}^{(m)}=f_{j k}, \quad j=1, \ldots, m_{0} \\
\sum_{m=1}^{p} \lambda_{m}^{r_{j}} C_{k}^{(m)}=f_{j k}, \quad j=m_{0}+1, \ldots, m_{1}
\end{gathered}
$$

где (учитьвая, что $r_{m_{0}+1}-1=r_{m_{0}}$ )

$$
f_{j k}=\left\{\begin{array}{c}
-\left.\left(\frac{\partial^{r_{j}} v_{k-r_{m_{0}}+r_{j}}}{\partial t^{r_{j}}}+\sum_{m=1}^{r_{j}} B_{j m} \frac{\partial^{r_{j}-m} v_{k-r_{m_{0}}+r_{j}-m}}{\partial t^{r_{j}-m}}\right)\right|_{t=0}, j=1, \ldots, m_{0}-1 ; \\
\delta\left(k+r_{m_{0}}-r_{j}\right) f_{j}-\left.B_{j} w_{k+r_{m_{0}}-r_{j}}\right|_{\Gamma} \\
-\left.\left(\frac{\partial^{r_{j}} \bar{v}_{k}}{\partial t^{r_{j}}}+\sum_{m=1}^{r_{j}} B_{j m} \frac{\partial^{r_{j}-m} v_{k-m}}{\partial t^{r_{j}-m}}\right)\right|_{t=0}, j=m_{0}+1, \ldots, m_{1}, \\
f_{m_{0} k}=-\left.\left(\frac{\partial^{r_{m_{0}} \bar{v}_{k}}}{\partial t^{r_{m_{0}}}}+\sum_{m=1}^{r_{m_{0}}} B_{m_{0} m} \frac{\partial^{r_{m_{0}}-m_{2}} v_{k-m}}{\partial t^{r_{m_{0}}-m}}\right)\right|_{t=0}
\end{array}\right.
$$

выражаются через уже известные функции. При этом ранг системы (5.8) равен $q$, а ее совместность обусловлена равенствами

$$
f_{i_{\alpha} k}-(-1)^{(p-1) N_{\alpha}}\left(\frac{a}{l}\right)^{N_{\alpha}} f_{j_{\alpha} k}=0, \quad \alpha=1, \ldots, p-q
$$

имеющими место по предположению индукции (при $k=1$ они вытекают из (4.5)). 
Далее, аналогично (4.5) из (2.11) имеем

$$
\begin{aligned}
& \left.\delta(\alpha-1) B_{m_{0}+1} w_{k}\right|_{\Gamma}+\left.\left(\frac{\partial^{r_{i \alpha}} v_{k+1}}{\partial t^{r_{i_{\alpha}}}}-(-1)^{(p-1) N_{\alpha}}\left(\frac{a}{l}\right)^{N_{\alpha}} \frac{\partial^{r_{j_{\alpha}}} v_{k+1}}{\partial t^{r_{j_{\alpha}}}}\right)\right|_{t=0} \\
& +\left(B_{i_{\alpha} 1}-(-1)^{(p-1) N_{\alpha}}\left(\frac{a}{l}\right)^{N_{\alpha}} B_{j_{\alpha} 1}\right) \sum_{m=1}^{p} \lambda_{m}^{r_{i_{\alpha}}-1} C_{k}^{(m)}=g_{k \alpha}^{\prime}, \quad \alpha=1, \ldots, p-q,
\end{aligned}
$$

где

$$
\begin{aligned}
g_{k \alpha}^{\prime}= & (1-\delta(\alpha-1))\left(\delta\left(k+i_{1}-i_{\alpha}\right) f_{i_{\alpha}}-B_{i_{\alpha}} w_{k+i_{1}-i_{\alpha}}\right) \\
& -\left.\left(B_{i_{\alpha} 1} \frac{\partial^{r_{i_{\alpha}}-1} \bar{v}_{k}}{\partial t^{r_{i_{\alpha}}}-1}+\sum_{m=2}^{r_{i_{\alpha}}} B_{i_{\alpha} m} \frac{\partial^{r_{i_{\alpha}}}-m}{\partial t^{r_{i_{\alpha}}}-m}\right)\right|_{t=0} \\
& -(-1)^{(p-1) N_{\alpha}}\left(\frac{a}{l}\right)^{N_{\alpha}}\left(\delta\left(k+i_{1}-j_{\alpha}\right) f_{j_{\alpha}}-B_{j_{\alpha}} w_{k+i_{1}-j_{\alpha}}\right. \\
& \left.-\left.\left(B_{j_{\alpha} 1} \frac{\partial^{r_{j_{\alpha}}}-1}{\partial t^{r_{j_{\alpha}}}-1}+\sum_{m=2}^{r_{j_{\alpha}}} B_{j_{\alpha} m} \frac{\partial^{r_{j_{\alpha}}-m} v_{k-m+1}}{\partial t^{r_{j_{\alpha}}}-m}\right)\right|_{t=0}\right)
\end{aligned}
$$

также выражаются через известные функции. Посредством последовательного интегрирования уравнения $M_{0} v_{k+1}=-\sum_{j=1}^{k+1} M_{j} v_{k-j+1}$ находим

$$
\begin{aligned}
& a \frac{\partial^{n_{\alpha m}+2 p} v_{k+1}}{\partial t^{n_{\alpha m}+2 p}}+(-1)^{p} l \frac{\partial^{n_{\alpha m}} v_{k+1}}{\partial t^{n_{\alpha m}}} \\
& =\left(-A_{1} \frac{\partial^{n_{\alpha m}+2 p-1}}{\partial t^{n_{\alpha m}+2 p-1}}-(-1)^{p} L_{1} \frac{\partial^{n_{\alpha m}-1}}{\partial t^{n_{\alpha m}-1}}-t\left(a_{1} \frac{\partial^{n_{\alpha m}+2 p}}{\partial t^{n_{\alpha m}+2 p}}+(-1)^{p} l_{1} \frac{\partial^{n_{\alpha m}}}{\partial t^{n_{\alpha m}}}\right)\right. \\
& \left.\quad+\left(2 m_{0}-n_{\alpha m}\right)\left(a_{1} \frac{\partial^{n_{\alpha m}+2 p-1}}{\partial t^{n_{\alpha m}+2 p-1}}+(-1)^{p} l_{1} \frac{\partial^{n_{\alpha m}-1}}{\partial t^{n_{\alpha m}-1}}\right)\right) \sum_{j=1}^{p} C_{k}^{(j)} e^{\lambda_{j} t}-F_{k \alpha m}
\end{aligned}
$$

где $n_{\alpha m}=r_{i_{\alpha}}+2 p m, 0 \leqslant m \leqslant N_{\alpha}-1$, а $F_{k \alpha m}$ - решение уравнения

$$
\frac{\partial^{2 m_{0}-n_{\alpha m}} F_{k \alpha m}}{\partial t^{2 m_{0}-n_{\alpha m}}}=M_{1} \bar{v}_{k}+\sum_{j=2}^{k+1} M_{j} v_{k-j+1},
$$

удовлетворяюшее условию (2.9). Теперь вычисления, аналогичные проделанным в п. 4.2 , приводят в силу $(5.9)$ к следующим соотношениям:

$$
\begin{gathered}
\left.B_{m_{0}+1} w_{k}\right|_{\Gamma}-T_{m_{0}+1} \sum_{m=1}^{p} \lambda_{m}^{r_{m_{0}}} C_{k}^{(m)}=g_{k 1}, \\
-T_{i_{\alpha}} \sum_{m=1}^{p} \lambda_{m}^{r_{i_{\alpha}}-1} C_{k}^{(m)}=g_{k \alpha}, \quad \alpha=2, \ldots, p-q,
\end{gathered}
$$

где

$$
g_{k \alpha}=g_{k \alpha}^{\prime}-\left.\sum_{m=0}^{N_{\alpha}-1}(-1)^{(p-1)(m+1)} \frac{a^{m}}{l^{m+1}} F_{k \alpha m}\right|_{t=0}, \quad \alpha=1, \ldots, p-q .
$$


В силу предположений индукции правые части равенств (5.7)-(5.11) принадлежат классу $C^{\infty}(\Gamma)$. Кроме того, соотношения (5.7) и (5.10) вместе с (2.2) приводят к краевой задаче

$$
\begin{aligned}
L w_{k}= & (-1)^{p-1} A w_{k-2 p},\left.\quad B_{j} w_{k}\right|_{\Gamma}=f_{j k}, \quad j=1, \ldots, m_{0}-1, \\
& \left.\left(B_{m_{0}+1}+T_{m_{0}+1} B_{m_{0}}\right) w_{k}\right|_{\Gamma}=g_{k 1}+T_{m_{0}+1} f_{m_{0} k} .
\end{aligned}
$$

Очередные коэффициенты асимптотического разложения определяются теперь по следующей схеме:

a) находим $w_{k} \in C^{\infty}(\bar{\Omega})$ как решение краевой задачи (5.12);

b) находим функции $h_{k \alpha} \in C^{\infty}(\Gamma)$ как решения уравнений

$$
T_{i_{\alpha}} h_{k \alpha}=-g_{k \alpha}, \quad \alpha=2, \ldots, p-q,
$$

на компактном многообразии $Г$;

c) исходя из $(5.7),(5.8)$ и $(5.11)$, определяем $C_{k}^{(1)}, \ldots, C_{k}^{(p)}$ как решение системы уравнений

$$
\begin{gathered}
\sum_{m=1}^{p} \lambda_{m}^{r_{i_{\alpha}}} C_{k}^{(m)}=f_{i_{\alpha} k}, \quad \alpha=1, \ldots, q, \\
\sum_{m=1}^{p} \lambda_{m}^{r_{m_{0}}} C_{k}^{(m)}=f_{m_{0} k}-\left.B_{m_{0}} w_{k}\right|_{\Gamma}, \\
\sum_{m=1}^{p} \lambda_{m}^{r_{i_{\alpha}}-1} C_{k}^{(m)}=h_{k \alpha}, \quad \alpha=2, \ldots, p-q
\end{gathered}
$$

(в силу (4.9) ее определитель отличен от нуля);

d) по формуле (5.6) находим $v_{k}(t, s)$, после чего, умножив $v_{k}$ на “срезающую" функцию, получаем коэффициент $v_{k}^{(\varepsilon)}$;

е) методом неопределенных коэффициентов находим $\bar{v}_{k+1}(t, s)$.

Описанное вьше построение коэффициентов $w_{k}$ и $v_{k}^{(\varepsilon)}$ обеспечивает выполнение соотношений $(2.2),(2.8),(2.9)$ и (2.11) для данного значения $k$. При этом соотношения (5.9) гарантируют совместность системы (5.8) на следуюшем шаге итерационного процесса.

Индукцией по $k$ теперь сразу устанавливается существование полного набора функций $w_{k}, v_{k}^{(\varepsilon)}$ класса $C^{\infty}(\bar{\Omega})$, удовлетворяющих всем требуемьм соотношениAM.

Шаг 3. Оценка остаточного члена. Согласно шагу 2 вблизи границы функция $v_{k}^{(\varepsilon)}=v_{k}(t, s)$ представляет собой квазимногочлен относительно $t=\rho / \varepsilon$ с показателями $\lambda_{1}(s) t, \ldots, \lambda_{p}(s) t$, причем $\operatorname{Re} \lambda_{j}(s)<0, j=1, \ldots, p$. Отсюда следует, что при любом натуральном $q$ существуют постоянные $C_{k q}$ и $C_{j k q}$, при которых

$$
\left\|v_{k}^{(\varepsilon)}\right\|_{H^{q}(\Omega)} \leqslant C_{k q} \varepsilon^{1 / 2-q}, \quad\left\|M_{j} v_{k}^{(\varepsilon)}\right\|_{H^{q}(\Omega)} \leqslant C_{j k q} \varepsilon^{1 / 2-q} .
$$

Пусть $\left\{w_{k}, v_{k}^{(\varepsilon)}\right\}$ - набор функций, полученный в результате описанного выше итерационного процесса. Введем обозначение

$$
u_{N, \varepsilon}=\sum_{k=0}^{N} \varepsilon^{k}\left(w_{k}+\varepsilon^{r_{m_{0}+1}-1} v_{k}^{(\varepsilon)}\right) \text {. }
$$

Тогда $z_{N, \varepsilon}=u_{\varepsilon}-u_{N, \varepsilon}$. 
Лемма. При любом $N \in \mathbb{N} \cup\{0\}$ имеет место оценка

$$
\left\|L_{\varepsilon} z_{N, \varepsilon}\right\|_{0} \leqslant C_{N} \varepsilon^{N+\min \left\{1, r_{m_{0}+1}-q_{0}+1 / 2\right\}}, \quad 0<\varepsilon \leqslant \varepsilon_{0} .
$$

ДокАЗАТЕльство. Действительно, в силу (1.2), (2.2) и (2.3) имеем

$$
\begin{aligned}
\left\|L_{\varepsilon} z_{N, \varepsilon}\right\|_{0}= & \left\|L_{\varepsilon} u_{N, \varepsilon}-h\right\|_{0}=\left\|\sum_{k=0}^{N} \varepsilon^{k}\left(L_{\varepsilon} w_{k}+\varepsilon^{r_{m_{0}+1}-1} L_{\varepsilon} v_{k}^{(\varepsilon)}-h\right)\right\|_{0} \\
\leqslant & \left\|\sum_{k=0}^{N} \varepsilon^{k}\left(\varepsilon^{2 p} A w_{k}+(-1)^{p} L w_{k}\right)-h\right\|_{0} \\
& +\left\|\sum_{k=0}^{N} \varepsilon^{k+r_{m_{0}+1}-1}\left(\sum_{j=0}^{N} \varepsilon^{j} M_{j} v_{k}^{(\varepsilon)}+R_{N, \varepsilon} v_{k}^{(\varepsilon)}\right)\right\|_{0} \\
\leqslant & \sum_{k=1}^{2 p} \varepsilon^{N+k}\left\|A w_{N+k-2 p}\right\|_{0}+\sum_{k=0}^{N} \varepsilon^{k+r_{m_{0}+1}-1}\left\|\sum_{j=0}^{k} M_{j} v_{k-j}^{(\varepsilon)}\right\|_{0} \\
& +\sum_{k=1}^{N} \varepsilon^{N+k+r_{m_{0}+1}-1}\left\|\sum_{j=k}^{N} M_{N+k-j} v_{j}^{(\varepsilon)}\right\|_{0} \\
& +\sum_{k=0}^{N} \varepsilon^{k+r_{m_{0}+1}-1}\left\|R_{N, \varepsilon} v_{k}^{(\varepsilon)}\right\|_{0} .
\end{aligned}
$$

Согласно (2.7) и (2.8)

$$
\sum_{j=0}^{k} M_{j} v_{k-j}^{(\varepsilon)}=0
$$

в некоторой полуокрестности $\Gamma$ (а также вне большей полуокрестности, поскольку там $\left.v_{k-j}^{(\varepsilon)} \equiv 0\right)$. Поэтому $\left\|\sum_{j=0}^{k} M_{j} v_{k-j}^{(\varepsilon)}\right\|_{0}$ убывает быстрее любой степени $\varepsilon$, так как $v_{k-j}^{(\varepsilon)}$ есть функция погранслоя. Кроме того, в силу (5.2) и (5.13)

$$
\left\|M_{N+k-j} v_{j}^{(\varepsilon)}\right\|_{0}=O\left(\varepsilon^{1 / 2-q_{0}}\right), \quad\left\|R_{N, \varepsilon} v_{k}^{(\varepsilon)}\right\|_{0}=O\left(\varepsilon^{N-q_{0}+3 / 2}\right) .
$$

Таким образом, окончательно получаем

$$
\begin{aligned}
\left\|L_{\varepsilon} z_{N, \varepsilon}\right\|_{0}= & O\left(\varepsilon^{N+1}\right)+o\left(\varepsilon^{N+r_{m_{0}+1}}\right) \\
& +O\left(\varepsilon^{N+r_{m_{0}+1}-q_{0}+1 / 2}\right)+O\left(\varepsilon^{N+r_{m_{0}+1}-q_{0}+1 / 2}\right) \\
= & O\left(\varepsilon^{N+\min \left\{1, r_{m_{0}+1}-q_{0}+1 / 2\right\}}\right) .
\end{aligned}
$$

Лемма доказана.

Пусть $N_{0}=N+k_{0}+q_{0}+r_{m_{1}}-r_{m_{0}+1}+1$, где $k_{0}$ определяется неравенством (5.1). Из построения коэффициентов асимптотического разложения видно, что

$$
\left.B_{j} u_{N_{0}, \varepsilon}\right|_{\Gamma}= \begin{cases}f_{j}+\varepsilon^{N_{0}+1} \sum_{k=0}^{k_{1}-1} \varepsilon^{k} h_{j k}^{(N)}, & j=1, \ldots, m_{0} ; \\ f_{j}+\varepsilon^{N_{0}+r_{m_{0}+1}-r_{j}} \sum_{k=0}^{k_{1}-r_{m_{0}+1}+r_{j}} \varepsilon^{k} h_{j k}^{(N)}, & j=m_{0}+1, \ldots, m_{1},\end{cases}
$$


где $k_{1}=\max \left\{2 p, r_{m_{0}+1}-1\right\}$, а $h_{j k}^{(N)}-$ функции класса $C^{\infty}(\Gamma)$. Отсюда следует, что сушествуют функции $w_{k}^{(N)} \in C^{\infty}(\bar{\Omega}), 0 \leqslant k \leqslant k_{1}-r_{m_{0}+1}+r_{m_{1}}$, такие, что

$$
z_{N, \varepsilon}^{\prime} \equiv z_{N, \varepsilon}+\varepsilon^{N+k_{0}+q_{0}+1} \sum_{k=0}^{k_{1}-r_{m_{0}+1}+r_{m_{1}}} \varepsilon^{k} w_{k}^{(N)}
$$

удовлетворяет однородным граничным условиям (1.3). Тогда в силу (5.1)

$$
\left\|z_{N, \varepsilon}^{\prime}\right\|_{1} \leqslant C \varepsilon^{-k_{0}}\left\|L_{\varepsilon} z_{N, \varepsilon}^{\prime}\right\|_{0}, \quad 0<\varepsilon \leqslant \varepsilon_{0} .
$$

Применив доказанную выше лемму, окончательно получим

$$
\begin{aligned}
& \left\|z_{N, \varepsilon}\right\|_{1} \leqslant\left\|z_{N_{0}, \varepsilon}\right\|_{1}+\left\|z_{N, \varepsilon}-z_{N_{0}, \varepsilon}\right\|_{1} \\
& \leqslant\left\|z_{N, \varepsilon}^{\prime}\right\|_{1}+\varepsilon^{N+k_{0}+q_{0}+1}\left\|_{\sum_{1}-k_{1}-r_{m_{0}+1}+r_{m_{1}}}^{k^{k}} w_{k}^{(N)}\right\|_{1}+\left\|u_{N_{0}, \varepsilon}-u_{N, \varepsilon}\right\|_{1} \\
& \leqslant C \varepsilon^{-k_{0}}\left\|L_{\varepsilon} z_{N, \varepsilon}^{\prime}\right\|_{0}+\varepsilon^{N+k_{0}+q_{0}+1} \sum_{k=0}^{k_{1}-r_{m_{0}+1}+r_{m_{1}}} \varepsilon^{k}\left\|w_{k}^{(N)}\right\|_{1} \\
& +\sum_{k=N+1}^{N_{0}} \varepsilon^{k}\left\|w_{k}+\varepsilon^{r_{m_{0}+1}-1} v_{k}^{(\varepsilon)}\right\|_{1} \\
& \leqslant C \varepsilon^{-k_{0}}\left\|L_{\varepsilon} z_{N, \varepsilon}\right\|_{0}+C \varepsilon^{N+q_{0}+1} \sum_{k=0}^{k_{1}-r_{m_{0}+1}+r_{m_{1}}} \varepsilon^{k}\left\|L_{\varepsilon} w_{k}^{(N)}\right\|_{0} \\
& +\varepsilon^{N+k_{0}+q_{0}+1} \sum_{k=0}^{k_{1}-r_{m_{0}+1}+r_{m_{1}}} \varepsilon^{k}\left\|w_{k}^{(N)}\right\|_{1} \\
& +\sum_{k=N+1}^{N_{0}} \varepsilon^{k}\left\|w_{k}\right\|_{1}+C \sum_{k=N+1}^{N_{0}} \varepsilon^{k+r_{m_{0}+1}-1}\left\|v_{k}^{(\varepsilon)}\right\|_{H^{q_{1}}(\Omega)} \\
& =O\left(\varepsilon^{N+r_{m_{1}}+\min \left\{q_{0}-r_{m_{0}+1}+2,3 / 2\right\}}\right)+O\left(\varepsilon^{N+q_{0}+1}\right) \\
& +O\left(\varepsilon^{N+k_{0}+q_{0}+1}\right)+O\left(\varepsilon^{N+1}\right)+O\left(\varepsilon^{N+r_{m_{0}+1}-q_{1}+1 / 2}\right) \\
& =O\left(\varepsilon^{N+\min \left\{1, r_{m_{0}+1}-q_{1}+1 / 2\right\}}\right) \text {, }
\end{aligned}
$$

что и завершает доказательство теоремы.

ЗАмечАниЕ 1. Если ранг системы (5.8) равен $p-1$, то условие 2 ) из формулировки теоремы, очевидно, исчезает. При этом также из схемы очередного шага итерационного процесса выпадает п. b).

ЗАмечАниЕ 2. Формально мы не требовали единственности решения вырожденной задачи. Тем не менее в условиях теоремы она всегда имеет место, поскольку, как известно, коэффициенты регулярной части асимптотического разложения определяются единственным образом. Следовательно, равномерная разрешимость задачи (1.2), (1.3) обеспечивает единственность решения вырожденной задачи (при условии, что последнее существует). 
ЗАмечАНИЕ 3 . При $n=1$ вместо умножения $v_{k}$ на “срезающую" функцию можно в качестве $v_{k}^{(\varepsilon)}$ взять сумму функций погранслоя, полученных вблизи правого и левого концов отрезка, на котором рассматривается краевая задача, поскольку при этом коэффициенты $v_{k}^{(\varepsilon)}$ изменятся на величины, убывающие быстрее любой степени $\varepsilon$.

ТЕОРЕМа 2. Пусть порядки граничных операторов (1.3) удовлетворяют условию (4.9), причем

1) $r_{m_{0}+1} \in J_{2}, r_{m_{0}+1}>r_{m_{0}}+1$;

2) для всех $f \in C^{\infty}(\Gamma)$ уравнения (5.3) при $k=1, \ldots, p-q$ разрешимь в классе $C^{\infty}(\Gamma)$

3) вырохсденная задача (1.4), (1.5) разрешима;

4) задача (1.2), (1.3) равномерно разрешима.

Тогда при любом $N \in \mathbb{N} \cup\{0\}$ решение задачи (1.2), (1.3) допускает представление в виде (5.4) с коэффиииентами класса $C^{\infty}(\bar{\Omega})$, причем остаточный член удовлетворяет оценке (5.5).

Ее доказательство практически повторяет предыдущее. Поэтому ограничимся схемой начального шага итерационного процесса.

a) Определяем $w_{0}$ как решение вырожденной краевой задачи.

b) Находим функцию $g \in C^{\infty}(\Gamma)$, удовлетворяющую уравнению

$$
T_{m_{0}+1} g=\left.B_{m_{0}+1} w_{0}\right|_{\Gamma}-f_{m_{0}+1}
$$

с) Исходя из $(4.10)$ и $(4.12)$, определяем $C_{0}^{(1)}, \ldots, C_{0}^{(p)}$ как решение системы уравнений

$$
\begin{gathered}
\sum_{m=1}^{p} \lambda_{m}^{r_{i_{k}}} C_{0}^{(m)}=0, \sum_{m=1}^{p} \lambda_{m}^{r_{m_{0}+1}-1} C_{0}^{(m)}=g, \sum_{m=1}^{p} \lambda_{m}^{r_{i_{\alpha}}-1} C_{0}^{(m)}=0, \\
k=1,2, \ldots, p, \quad \alpha=2, \ldots, p-q .
\end{gathered}
$$

d) По формуле (3.3) находим $v_{0}(t, s)$ и умножением на “срезающую” функцию строим коэффициент $v_{0}^{(\varepsilon)}$.

е) Находим частное решение $\bar{v}_{1}(t, s)$.

При выполнении условий теоремы 1 или теоремы 2 имеет место следующая ситуация. Изменение правых частей определяющих граничных условий влечет изменение того же порядка (относительно $\varepsilon$ ) решения задачи (1.2), (1.3). В свою очередь, при изменении граничных условий, не входящих в число определяющих, изменение решения $u_{\varepsilon}$ имеет порядок малости $O(\varepsilon)$. Таким образом, определяющие граничные условия - в точности те граничные условия, относительно которых решение задачи не является асимптотически (при $\varepsilon \rightarrow 0)$ устойчивым.

5.3. ПримеР 1. Рассмотрим на отрезке $[0,1]$ краевую задачу (1.6). Применим к ней описанный выше итерационный процесс нахождения коэффициентов асимптотического разложения. Поскольку задача инвариантна относительно замены $x$ на $1-x$, достаточно рассматривать граничные условия в точке $x=0$. 
В данном случае имеем

$$
\begin{gathered}
t=\frac{x}{\varepsilon}, \quad M_{0}=\frac{d^{8}}{d t^{8}}+4 \frac{d^{4}}{d t^{4}}, \quad M_{j}=0, \quad j=1,2, \ldots, \\
\lambda_{1,2}=-1 \pm i, \quad B_{m_{0}}=B_{2}=\frac{d}{d x}, \quad B_{j k}=0, \quad j=1,2,3,4, \quad k=1,2, \ldots
\end{gathered}
$$

Вырожденная задача

$$
4 w_{0}^{(4)}=0,\left.\quad w_{0}\right|_{\Gamma}=0,\left.\quad w_{0}^{\prime \prime}\right|_{\Gamma}=1
$$

была получена в конще $\S 4$. Решая ее, находим $w_{0}=\left(x^{2}-x\right) / 2$. Поскольку $w_{0}^{\prime}(0)=$ $-\frac{1}{2}$, согласно $(4.2)$ и (4.3) имеем систему уравнений

$$
\lambda_{1} C_{0}^{(1)}+\lambda_{2} C_{0}^{(2)}=\frac{1}{2}, \quad \lambda_{1}^{2} C_{0}^{(1)}+\lambda_{2}^{2} C_{0}^{(2)}=0
$$

откуда

$$
C_{0}^{(1)}=C_{0}^{(2)}=-\frac{1}{4}, \quad v_{0}=-\frac{1}{4} e^{-t}\left(e^{i t}+e^{-i t}\right)=-\frac{1}{2} e^{-t} \cos t=-\frac{1}{2} e^{-x / \varepsilon} \cos \frac{x}{\varepsilon} .
$$

В окрестности точки $x=1$ соответственно имеем

$$
v_{0}=-\frac{1}{2} e^{-(1-x) / \varepsilon} \cos \frac{1-x}{\varepsilon}
$$

Поэтому $\left.v_{0}^{(\varepsilon)}\right|_{\Gamma}=-\frac{1}{2}$ и краевая задача для $w_{1}$ принимает вид

$$
4 w_{1}^{(4)}=0,\left.\quad w_{1}\right|_{\Gamma}=\frac{1}{2},\left.\quad w_{1}^{\prime \prime}\right|_{\Gamma}=0
$$

откуда $w_{1}=\frac{1}{2}$. Далее, согласно (2.8) $M_{0} v_{1}=0$, откуда $v_{1}=C_{1}^{(1)} e^{\lambda_{1} t}+C_{1}^{(2)} e^{\lambda_{2} t}$. Следовательно, поскольку $w_{1}^{\prime}=0$, имеем систему уравнений

$$
\lambda_{1} C_{1}^{(1)}+\lambda_{2} C_{1}^{(2)}=0, \quad \lambda_{1}^{2} C_{1}^{(1)}+\lambda_{2}^{2} C_{1}^{(2)}=0,
$$

в силу которой $C_{1}^{(1)}=C_{1}^{(2)}=0, v_{1}=0$. На следующих шагах итерационного процесса получаются соотношения между коэффициентами разложения с нулевыми правыми частями, вследствие чего $w_{k}=0, v_{k}=0$ при $k \geqslant 2$. Таким образом,

$$
\begin{gathered}
w_{0}=\frac{x^{2}-x}{2}, \quad w_{1}=\frac{1}{2}, \quad w_{k}=0, \quad k=2,3, \ldots, \\
v_{0}^{(\varepsilon)}=-\frac{1}{2}\left(e^{-x / \varepsilon} \cos \frac{x}{\varepsilon}+e^{-(1-x) / \varepsilon} \cos \frac{1-x}{\varepsilon}\right), \quad v_{k}^{(\varepsilon)}=0, \quad k=1,2, \ldots
\end{gathered}
$$

(см. замечание 3 ). 
Все это позволяет сделать предположение о том, что решение задачи (1.6) имеет следуюшее асимптотическое представление:

$$
u_{\varepsilon} \sim \frac{x^{2}-x}{2}+\frac{\varepsilon}{2}\left(1-e^{-x / \varepsilon} \cos \frac{x}{\varepsilon}-e^{-(1-x) / \varepsilon} \cos \frac{1-x}{\varepsilon}\right)
$$

(поскольку равномерная разрешимость не была установлена, мы не можем здесь применить теорему 1). Непосредственное исследование формулы для $u_{\varepsilon}(x)$, приведенной в $\S 1$, показывает, что такое представление действительно имеет место, причем

$$
u_{\varepsilon}=\frac{x^{2}-x}{2}+\frac{\varepsilon}{2}\left(1-e^{-x / \varepsilon} \cos \frac{x}{\varepsilon}-e^{-(1-x) / \varepsilon} \cos \frac{1-x}{\varepsilon}\right)+o\left(e^{(\delta-1) / \varepsilon}\right),
$$

где $\delta$ - произвольное положительное число. В данном случае асимптотическое разложение решения краевой задачи состоит из конечного числа членов.

ПримеР 2. Рассмотрим в ограниченной области $\Omega \subset \mathbb{R}^{2}$ краевую задачу

$$
\begin{gathered}
L_{\varepsilon} u_{\varepsilon} \equiv \varepsilon^{4} \Delta^{4} u_{\varepsilon}+\Delta^{2} u_{\varepsilon}+u_{\varepsilon}=h \\
\left.\frac{\partial u_{\varepsilon}}{\partial \nu}\right|_{\Gamma}=f_{1},\left.\quad \Delta u_{\varepsilon}\right|_{\Gamma}=f_{2},\left.\quad \frac{\partial \Delta u_{\varepsilon}}{\partial \nu}\right|_{\Gamma}=f_{3},\left.\quad \frac{\partial \Delta^{3} u_{\varepsilon}}{\partial \nu}\right|_{\Gamma}=f_{4},
\end{gathered}
$$

где $\nu$ - направление внутренней нормали к Г. Здесь $r_{1}=1, r_{2}=2, r_{3}=3, r_{4}=7$.

Пусть $u \in C^{\infty}(\bar{\Omega})$ удовлетворяет однородным граничным условиям (5.15). Положим

$$
\|u\|_{0}=\|u\|_{L^{2}(\Omega)}, \quad\|u\|_{1}=\left(\int_{\Omega}\left(\left(\Delta^{2} u\right)^{2}+(\Delta u)^{2}+u^{2}\right) d x\right)^{1 / 2} .
$$

По формуле Грина имеем

$$
\begin{aligned}
\int_{\Omega} u L_{\varepsilon} u d x= & \int_{\Omega}\left(\varepsilon^{4}\left(\Delta^{2} u\right)^{2}+(\Delta u)^{2}+u^{2}\right) d x \\
& +\int_{\Gamma}\left(\frac{\partial u}{\partial \nu}\left(\varepsilon^{4} \Delta^{3} u+\Delta u\right)-\varepsilon^{4} \Delta u \frac{\partial \Delta^{2} u}{\partial \nu}\right. \\
& \left.+\frac{\partial \Delta u}{\partial \nu}\left(\varepsilon^{4} \Delta^{2} u-u\right)-\varepsilon^{4} u \frac{\partial \Delta^{3} u}{\partial \nu}\right) d s \\
= & \int_{\Omega}\left(\varepsilon^{4}\left(\Delta^{2} u\right)^{2}+(\Delta u)^{2}+u^{2}\right) d x \geqslant \varepsilon^{4}\|u\|_{1}^{2}, \quad 0<\varepsilon \leqslant 1 .
\end{aligned}
$$

Поскольку $\|u\|_{0} \leqslant\|u\|_{1}$, отсюда получаем

$$
\|u\|_{1} \leqslant \varepsilon^{-4}\left\|L_{\varepsilon} u\right\|_{0}, \quad 0<\varepsilon \leqslant 1
$$

Легко также проверить, что задача $(5.14)$, (5.15) удовлетворяет условию Шапиро-Лопатинского и является самосопряженной относительно формулы Грина, в силу чего неравенство (5.16) обеспечивает ее равномерную разрешимость. 
Далее, в координатах $(\rho, s)(s-$ длина дуги вдоль Г)

$$
\Delta=\frac{\partial^{2}}{\partial \rho^{2}}-\frac{\varkappa(s)}{1-\rho \varkappa(s)} \frac{\partial}{\partial \rho}+\frac{1}{(1-\rho \varkappa(s))^{2}} \frac{\partial^{2}}{\partial s^{2}}+\frac{\varkappa^{\prime}(s)}{(1-\rho \varkappa(s))^{3}} \frac{\partial}{\partial s},
$$

где $\varkappa(s)-$ кривизна Г в точке $s$. Следовательно, граничные операторы в (5.15) нормированы. Применительно к рассматриваемой задаче формула (4.8) примет вид

$$
T_{3}=\frac{L_{1}}{l}-\frac{A_{l}}{l} \frac{l}{a}+\frac{a_{1}}{a}-\frac{l_{1}}{l}-B_{31}+\frac{a}{l} B_{41} \frac{l}{a} .
$$

Из (5.17) следует, что $a=l=1, a_{1}=l_{1}=0, A_{1}=-4 \varkappa(s), L_{1}=-2 \varkappa(s)$, $B_{31}=-\varkappa(s), B_{41}=-3 \varkappa(s)$. Подставив все это в (5.18), получим, что $T_{3}=0$, в силу чего вырожденная задача запишется следующим образом:

$$
\Delta^{2} w_{0}+w_{0}=h,\left.\quad \frac{\partial w_{0}}{\partial \nu}\right|_{\Gamma}=f_{1},\left.\quad \frac{\partial \Delta w_{0}}{\partial \nu}\right|_{\Gamma}=f_{3} .
$$

Эта задача также самосопряжена относительно формулы Грина и ее разрешимость доказывается точно так же, как и разрешимость задачи (5.14), (5.15).

Таким образом, условия теоремы 1 выполнены, в силу чего

$$
u_{\varepsilon}=\sum_{k=0}^{N} \varepsilon^{k}\left(w_{k}+\varepsilon^{2} v_{k}^{(\varepsilon)}\right)+z_{N, \varepsilon}
$$

причем (поскольку здесь $q_{1}=4$ )

$$
\left\|z_{N, \varepsilon}\right\|_{1} \leqslant C_{N} \varepsilon^{N-1 / 2}
$$

\section{§6. Геометрическая интерпретация понятия равномерной разрешимости в соболевских пространствах}

6.1. Для получения результатов этого параграфа нет необходимости предполагать оператор $L$ эллиптическим. Поэтому здесь $A$ будет эллиптическим оператором порядка $2 m$, а $L$ - дифференциальньм оператором порядка $2 m-p$. Кроме того, краевую задачу

$$
L_{\varepsilon} u_{\varepsilon} \equiv \varepsilon^{p} A u_{\varepsilon}+L u_{\varepsilon}=h,\left.\quad B_{j} u_{\varepsilon}\right|_{\Gamma}=f_{j}, \quad j=1,2, \ldots, m
$$

(где, по-прежнему, $r_{1}<r_{2}<\cdots<r_{m} \leqslant 2 m-1$ ), будем рассматривать в пространстве размерности больше или равной 2 с тем, чтобы применить классическую теорию эллиптических краевых задач [1]. Введем также обозначение

$$
U=\left\{u \in C^{\infty}(\bar{\Omega}):\left.B_{j} u\right|_{\Gamma}=f_{j}, j=1,2, \ldots, m\right\} .
$$

Пусть задача (6.1) удовлетворяет условию Шапиро-Лопатинского и, кроме того, допускает (при малых $\varepsilon$ ) единственное решение. Положим

$$
\mathscr{A}_{\varepsilon} u=\left(L_{\varepsilon} u,\left.B_{1} u\right|_{\Gamma}, \ldots,\left.B_{m} u\right|_{\Gamma}\right)
$$


Тогда [1] оператор

$$
\mathscr{A}_{\varepsilon}: H^{q}(\Omega) \rightarrow H^{q-2 m}(\Omega) \times \prod_{j=1}^{m} H^{q-r_{j}-1 / 2}(\Gamma), \quad q \geqslant 2 m
$$

непрерывен, инъективен, его образ $\operatorname{Im} \mathscr{A}_{\varepsilon}$ замкнут, причем codim $\operatorname{Im} \mathscr{A}_{\varepsilon}<\infty$. Следовательно, подпространство $\operatorname{Im} \mathscr{A}_{\varepsilon}$ само является банаховым пространством. По теореме Банаха об открытом отображении обратный оператор

$$
\mathscr{A}_{\varepsilon}^{-1}: \operatorname{Im} \mathscr{A}_{\varepsilon} \rightarrow H^{q}(\Omega)
$$

ограничен, т.е.

$$
\|u\|_{H^{q}(\Omega)} \leqslant C(\varepsilon)\left\|\mathscr{A}_{\varepsilon} u\right\|_{H^{q-2 m}(\Omega) \times \prod_{j=1}^{m} H^{q-r_{j}-1 / 2}(\Gamma)}, \quad 0<\varepsilon \leqslant \varepsilon_{0} .
$$

В частности, для $u \in U$ имеем

$$
\|u\|_{H^{q}(\Omega)} \leqslant C(\varepsilon)\left\|L_{\varepsilon} u\right\|_{H^{q-2 m}(\Omega)}, \quad 0<\varepsilon \leqslant \varepsilon_{0} .
$$

Условие равномерной разрешимости (относительно $H^{q-2 m}(\Omega)$ и $H^{q}(\Omega)$ ) дополнительно предполагает, что $\operatorname{Im} \mathscr{A}_{\varepsilon}=H^{q-2 m}(\Omega) \times \prod_{j=1}^{m} H^{q-r_{j}-1 / 2}(\Gamma)$ (поскольку подпространство бесконечно дифференцируемых функций плотно в соболевских пространствах), а $C(\varepsilon)$ в неравенстве (6.2) допускает степенной рост, т.е.

$$
\|u\|_{H^{q}(\Omega)} \leqslant C \varepsilon^{-k_{0}}\left\|L_{\varepsilon} u\right\|_{H^{q-2 m}(\Omega)}, \quad 0<\varepsilon \leqslant \varepsilon_{0}
$$

Здесь равномерная разрешимость задачи (6.1) будет рассматриваться лишш относительно пространств $H^{q-2 m}(\Omega)$ и $H^{q}(\Omega)$, где $q$ - фиксированное целое число $q \geqslant 2 m$. Поэтому в дальнейшем слова “относительно $H^{q-2 m}(\Omega)$ и $H^{q}(\Omega)$ ” будут опускаться. В соответствии с этим будем обозначать

$$
\|\cdot\|_{0}=\|\cdot\|_{H^{q-2 m}(\Omega)}, \quad\|\cdot\|_{1}=\|\cdot\|_{H^{q}(\Omega)} .
$$

Отметим также, что равномерно разрешимая краевая задача, удовлетворяюшая условию Шапиро-Лопатинского, заведомо имеет нулевой индекс.

Лемма. Пусть $k_{0}$ - челое число, $k_{0} \geqslant p$, задача (6.1) удовлетворяет условию Шапиро-Лопатинского, имеет нулевой индекс и при $\varepsilon=\varepsilon_{0}$ допускает единственное решение. Для того чтобъ на интервале $\left(0, \varepsilon_{0}\right]$ она бъла равномерно разрешимой с показателем $k_{0}$, необходимо и достаточно, чтобы существовала постоянная $C_{0}>0$, при которой для всех $u \in U$ имеет место неравенство

$$
\|A u\|_{0} \leqslant C_{0} \varepsilon^{-k_{0}}\left\|L_{\varepsilon} u\right\|_{0}, \quad 0<\varepsilon \leqslant \varepsilon_{0} .
$$


ДоказАТЕльство. В силу неравенства $\|A u\|_{0} \leqslant$ const $\|u\|_{1}$ необходимость условия (6.4) очевидна. Докажем его достаточность.

Пусть $u$ - произвольная функция из $U$. Через $u^{(\varepsilon)}$ обозначим решение краевой задачи

$$
L_{\varepsilon_{0}} u^{(\varepsilon)}=L_{\varepsilon} u,\left.\quad B_{j} u^{(\varepsilon)}\right|_{\Gamma}=0, \quad j=1,2, \ldots, m .
$$

Сушествование решения следует из единственности, поскольку индекс задачи равен нулю. При этом $u^{(\varepsilon)} \in C^{\infty}(\bar{\Omega})$, так как этому классу принадлежит правая часть уравнения. Положим $v_{\varepsilon}=u-u^{(\varepsilon)}$. Тогда

$$
L_{\varepsilon_{0}} v_{\varepsilon}=L_{\varepsilon_{0}} u-L_{\varepsilon_{0}} u^{(\varepsilon)}=L_{\varepsilon_{0}} u-L_{\varepsilon} u=\left(\varepsilon_{0}^{p}-\varepsilon^{p}\right) A u,
$$

откуда

$$
\left\|L_{\varepsilon_{0}} v_{\varepsilon}\right\|_{0}=\left(\varepsilon_{0}^{p}-\varepsilon^{p}\right)\|A u\|_{0}, \quad 0<\varepsilon \leqslant \varepsilon_{0} .
$$

Так как $u \in U, u^{(\varepsilon)} \in U$, то $v_{\varepsilon} \in U$. Из (6.2) и (6.4) теперь получаем

$$
\begin{gathered}
\left\|v_{\varepsilon}\right\|_{1} \leqslant C\left(\varepsilon_{0}\right)\left\|L_{\varepsilon_{0}} v_{\varepsilon}\right\|_{0}=C\left(\varepsilon_{0}\right)\left(\varepsilon_{0}^{p}-\varepsilon^{p}\right)\|A u\|_{0} \\
\leqslant C_{0} C\left(\varepsilon_{0}\right) \varepsilon^{p-k_{0}}\left(\left(\frac{\varepsilon_{0}}{\varepsilon}\right)^{p}-1\right)\left\|L_{\varepsilon} u\right\|_{0}, \\
\left\|u^{(\varepsilon)}\right\|_{1} \leqslant C\left(\varepsilon_{0}\right)\left\|L_{\varepsilon_{0}} u^{(\varepsilon)}\right\|_{0}=C\left(\varepsilon_{0}\right)\left\|L_{\varepsilon} u\right\|_{0} .
\end{gathered}
$$

Можно считать, что $C_{0} \varepsilon_{0}^{p-k_{0}} \geqslant 1$. Тогда в силу неравенства $k_{0} \geqslant p$ имеем

$$
\begin{aligned}
\|u\|_{1} & =\left\|u^{(\varepsilon)}+v_{\varepsilon}\right\|_{1} \leqslant\left\|u^{(\varepsilon)}\right\|_{1}+\left\|v_{\varepsilon}\right\|_{1} \\
& \leqslant C_{0} C\left(\varepsilon_{0}\right) \varepsilon^{p-k_{0}}\left\|L_{\varepsilon} u\right\|_{0}+C_{0} C\left(\varepsilon_{0}\right) \varepsilon^{p-k_{0}}\left(\left(\frac{\varepsilon_{0}}{\varepsilon}\right)^{p}-1\right)\left\|L_{\varepsilon} u\right\|_{0} \\
& =C_{0} C\left(\varepsilon_{0}\right) \varepsilon^{-k_{0}} \varepsilon_{0}^{p}\left\|L_{\varepsilon} u\right\|_{0}, \quad 0<\varepsilon \leqslant \varepsilon_{0} .
\end{aligned}
$$

Полагая $C=C_{0} C\left(\varepsilon_{0}\right) \varepsilon_{0}^{p}$, приходим к неравенству (6.3), которое обеспечивает равномерную разрешимость задачи (6.1) с показателем $k_{0}$. Лемма доказана.

Эта лемма позволяет теперь оперировать только с нормой в пространстве $H^{q-2 m}(\Omega)$.

6.2. Через $(\cdot, \cdot)$ обозначим скалярное произведение в $H^{q-2 m}(\Omega)$, определяющее норму $\|\cdot\|_{0}$ :

$$
(u, v)=\int_{\Omega} \sum_{|\alpha| \leqslant q-2 m} \frac{\partial^{\alpha} u}{\partial x^{\alpha}} \frac{\partial^{\alpha} v}{\partial x^{\alpha}} d x .
$$

Теорема. Пусть краевая задача (6.1) әллиптична в смысле Шапиро-Лопатинского и имеет нулевой индекс. Для того чтобъ она была равномерно разрешимой с показателем $k_{0} \geqslant p$, необходимо и достаточно, чтобы выполнялись следующие условия:

1) $U \cap \operatorname{Ker} A \cap \operatorname{Ker} L=\{0\}$;

2) существуют положсительнье постоянные $C_{1} u C_{2}$, при которых для любой функции $u \in U$ выполняется хотя бы одно из неравенств

$$
\begin{gathered}
\|A u\|_{0} \leqslant C_{1}\|L u\|_{0} \\
(A u, L u) \geqslant-\|A u\|_{0}\|L u\|_{0}\left(1-C_{2}\left(\frac{\|L u\|_{0}}{\|A u\|_{0}}\right)^{2 k_{0} / p-2}\right) .
\end{gathered}
$$


ДоказАТЕльство. Предположим, что на интервале $\left(0, \varepsilon_{0}\right]$ имеет место равномерная разрешимость с показателем $k_{0} \geqslant p$. Необходимость условия 1$)$ тогда очевидна. Если $A u=0$, то имеет место неравенство (6.5), а при $L u=0$ - неравенство (6.6). Остается рассмотреть случай $A u \neq 0, L u \neq 0, u \in U$.

Введем следуюшие обозначения:

$$
\rho=\frac{\|A u\|_{0}}{\|L u\|_{0}}, \quad \cos \varphi=\frac{(A u, L u)}{\|A u\|_{0}\|L u\|_{0}}, \quad 0 \leqslant \varphi \leqslant \pi
$$

(другими словами, $\varphi$ является углом между векторами $A u$ и $L u$ в гильбертовом пространстве $\left.H^{q-2 m}(\Omega)\right)$. Тогда (6.5) будет эквивалентно неравенству

$$
\rho \leqslant C_{1}
$$

а (6.6) - неравенству

$$
1+\cos \varphi \geqslant C_{2} \rho^{-2 k / p}
$$

где $k=k_{0}-p \geqslant 0$. Далее, в силу леммы имеет место неравенство (6.4) с не зависящей от $u$ постоянной $C_{0}>0$. Так как

$$
\begin{aligned}
\left\|L_{\varepsilon} u\right\|_{0}^{2}=\left(L_{\varepsilon} u, L_{\varepsilon} u\right) & =\varepsilon^{2 p}\|A u\|_{0}^{2}+2 \varepsilon^{p}(A u, L u)+\|L u\|_{0}^{2} \\
& =\|L u\|_{0}^{2}\left(\varepsilon^{2 p} \rho^{2}+2 \varepsilon^{p} \rho \cos \varphi+1\right),
\end{aligned}
$$

то (6.4) эквивалентно неравенству

$$
\rho^{2} \leqslant C_{0}^{2} \varepsilon^{-2 k_{0}}\left(\varepsilon^{2 p} \rho^{2}+2 \varepsilon^{p} \rho \cos \varphi+1\right)
$$

или

$$
\left(1-\left(\frac{\varepsilon^{k}}{C_{0}}\right)^{2}\right) \rho^{2}+2 \varepsilon^{-p} \rho \cos \varphi+\varepsilon^{-2 p} \geqslant 0, \quad 0<\varepsilon \leqslant \varepsilon_{0} .
$$

Теперь предположим, что выполнены условия 1) и 2) с фиксированными положительными постоянньми $C_{1}$ и $C_{2}$. Отсюда сразу вытекает единственность решения задачи (6.1) при $\varepsilon<C_{1}^{-1 / p}$. Следовательно (в силу леммы), условие равномерной разрешимости с показателем $k_{0}$ эквивалентно (6.4).

При $A u=0$ условие (6.4), очевидно, выполняется. Если $L u=0$, то $L_{\varepsilon} u=\varepsilon^{p} A u$ и опять имеет место (6.4) при $\varepsilon_{0}=1, k_{0}=p$ (и тем более при $k_{0} \geqslant p$ ), $C_{0}=1$. Если же $A u \neq 0, L u \neq 0$, то, как было показано выше, условие (6.4) эквивалентно (6.9) при $k=k_{0}-p$.

Таким образом, доказательство свелось к исследованию квадратного неравенства (6.9) с двумя параметрами. Это исследование показывает, что неравенство (6.9) вьполняется на некотором интервале $\left(0, \varepsilon_{0}\right]$ тогда и только тогда, когда существуют положительные постоянные $C_{1}$ и $C_{2}$, при которых $\rho$ и $\varphi$ удовлетворяют хотя бы одному из неравенств (6.7), (6.8), что и доказывает теорему. Полное ее доказательство приведено в депонированной работе [6]. 
ЗАМЕЧАнИЕ 1. Условие 1) тривиально и абсолютно необходимо для равномерной разрешимости задачи (6.1). Однако без него невозможно установить единственность решения. Основное содержание теоремы заключается в условии 2), которое в форме (6.7), (6.8) дает геометрическую интерпретацию понятия равномерной разрешимости.

ЗАмЕчАниЕ 2. Критерий равномерной разрешимости был доказан для показателей $k_{0} \geqslant p$. Без дополнительных условий на операторы $A$ и $L$ это ограничение неизбежно. Дело в том, что на подпространстве $U$ оператор $L$ может иметь нетривиальное ядро. Тогда для некоторой функции $u_{0} \in U, u_{0} \neq 0$, получим $\varepsilon^{p} A u_{0}=L_{\varepsilon} u_{0}$ и как следствие неравенство (6.4) при $k_{0}=p$. Поскольку при этом $A u_{0} \neq 0$ (условие 1$)$ ), то заменить этот показатель на меньший нельзя.

СЛЕДСТВИЕ. Пусть выполнены условия теоремы. Если при некотором $\varepsilon_{0}>0$ для всех $u \in U, u \neq 0$, вицолняется неравенство

$$
\left(L_{\varepsilon_{0}} u, L u\right)>0
$$

то краевая задача (6.1) равномерно разрешима с показателем $k_{0}=p$.

В самом деле, из (6.10) сразу вытекает, что $L u \neq 0$ и, значит, условие 1$)$ выполнено. Далее, в принятых вьше обозначениях (6.10) эквивалентно неравенству

$$
\rho \cos \varphi>-\varepsilon_{0}^{-p}
$$

Тогда из $\rho>C_{1}>0$ следует, что $\cos \varphi>-\varepsilon_{0}^{-p} / C_{1}$. Возьмем в качестве $C_{1}$ любое число больше $\varepsilon_{0}^{-p}$ и положим $C_{2}=1-\varepsilon_{0}^{-p} / C_{1}>0$. В результате получим, что при $\rho>C_{1}$ имеет место неравенство (6.8) с $k=0$, что соответствует $k_{0}=p$. Следовательно, условие 2) также вьполнено и задача (6.1) равномерно разрешима с показателем $p$.

Наиболее простой вид условие (6.10) принимает при $q=2 m$ :

$$
\int_{\Omega} L_{\varepsilon_{0}} u L u d x>0, \quad u \in U, \quad u \neq 0 .
$$

6.3. ПРИмеР. Рассмотрим краевую задачу

$$
\begin{gathered}
L_{\varepsilon} u_{\varepsilon} \equiv \varepsilon^{4} \Delta^{4} u_{\varepsilon}+\Delta^{2} u_{\varepsilon}=h \\
\left.u_{\varepsilon}\right|_{\Gamma}=f_{1},\left.\quad \Delta u_{\varepsilon}\right|_{\Gamma}=f_{2},\left.\quad \Delta^{2} u_{\varepsilon}\right|_{\Gamma}=f_{3},\left.\quad \Delta^{3} u_{\varepsilon}\right|_{\Gamma}=f_{4} .
\end{gathered}
$$

Задача (6.11), (6.12) удовлетворяет условию Шапиро-Лопатинского и является самосопряженной относительно формулы Грина, в силу чего ее индекс равен нулю. Пусть $u \in C^{\infty}(\bar{\Omega})$ удовлетворяет однородным граничным условиям (6.12). Применив формулу Грина, получим

$$
\int_{\Omega}\left(\Delta^{4} u+\Delta^{2} u\right) \Delta^{2} u d x=\int_{\Omega}\left(\left(\Delta^{3} u\right)^{2}+\left(\Delta^{2} u\right)^{2}\right) d x \geqslant 0
$$


Если при этом $u \not \equiv 0$, то имеет место строгое неравенство

$$
\int_{\Omega}\left(\Delta^{4} u+\Delta^{2} u\right) \Delta^{2} u d x>0
$$

поскольку задача

$$
\Delta^{2} u=0,\left.\quad u\right|_{\Gamma}=0,\left.\quad \Delta u\right|_{\Gamma}=0,
$$

имеет лиш тривиальное решение. Таким образом, условие (6.10) выполняется при $\varepsilon_{0}=1$ и задача $(6.11),(6.12)$ равномерно разрешима с показателем 4 :

$$
\|u\|_{H^{8}(\Omega)} \leqslant C \varepsilon^{-4}\left\|L_{\varepsilon} u\right\|_{L^{2}(\Omega)}, \quad 0<\varepsilon \leqslant \delta<1,
$$

для всех $u$, удовлетворяющих однородньм граничным условиям (6.12).

В рассматриваемом примере $r_{1}=0, r_{2}=2, r_{3}=4, r_{4}=6, q_{0}=0, q_{1}=8$, $r_{3} \not \equiv r_{4}(\bmod 4)$. Поэтому [5] при любом $N \in \mathbb{N} \cup\{0\}$ решение задачи $(6.11),(6.12)$ допускает представление в виде

$$
u_{\varepsilon}=\sum_{k=0}^{N} \varepsilon^{k}\left(w_{k}+\varepsilon^{4} v_{k}^{(\varepsilon)}\right)+z_{N, \varepsilon}
$$

причем остаточный член удовлетворяет оценке

$$
\left\|z_{N, \varepsilon}\right\|_{H^{8}(\Omega)} \leqslant C_{N} \varepsilon^{N+\min \left\{1, r_{3}-q_{1}+3 / 2\right\}}=C_{N} \varepsilon^{N-5 / 2} .
$$

\section{Список литературы}

1. Лионс Ж.-Л., Мадженес Э. Неоднородные граничные задачи и их приложения. Т. 1. М.: Мир, 1971.

2. Вишик М.И., Люстерник Л. А. Регулярное вырождение и пограничный слой для линейных дифференциальных уравнений с мальм параметром // УМН. 1957. Т. 12. №5. C. $3-122$.

3. Вазов В. Асимптотические разложения решений обыкновенных дифференциальных уравнений. М.: Мир, 1968.

4. Голопуз C. A. Об определяющих граничных условиях для эллиптических краевых задач с мальм параметром при старших производных // Труды МИРАН. 2002. Т. 236. С. 87-94.

5. Lin Zongchi. Asymptotic expression of the solution of general boundary value problem for higher order elliptic equation with perturbation both boundary and operator // Appl. Math. Mech. 1982. V. 3. № 1. P. 33-47.

6. Голопуз C.A. Критерий равномерной разрешимости эллиптических краевых задач с мальп параметром при старших производных // Деп. в ВИНИТИ. 20.11.90. № 5826-В90.

Владимирский государственнњй университет

Поступила в редакцию 04.06 .2002 